\title{
Novel heterocyclic $\alpha$-amino acids with sulfur-containing side-chains
}

\author{
Raymond C. F. Jones, ${ }^{\text {a }}$ Lisa J. Crumpling, ${ }^{\text {a }}$ and James N. Iley ${ }^{b}$ \\ ${ }^{a}$ Department of Chemistry, Loughborough University, Loughborough, Leics. LE11 3TU, UK \\ ${ }^{b}$ Department of Chemistry, Open University, Walton Hall, Milton Keynes MK7 6AA, UK \\ E-mail: r.c.f.jones@lboro.ac.uk
}

\section{Dedicated to Professor Siegfried Blechert on the occasion of his $65^{\text {th }}$ birthday}

\begin{abstract}
$\mathrm{S}$-Alkylation of an $\mathrm{N}$-protected cysteine ester with a range of $\omega$-iodoalkyl heterocycles affords 11 novel non-proteinogenic heterocyclic amino acids having a sulfur atom in the backboneheterocycle linker.
\end{abstract}

Keywords: Amino acid, heterocycle, cysteine, nucleobase

\section{Introduction}

Amongst the proteinogenic amino acids, there are only two examples of side chains carrying a heterocycle, namely histidine $\mathbf{1}$ and tryptophan $\mathbf{2}$ (Figure 1). On the other hand heterocyclic chemistry provides an enormous range of potential heterocyclic systems that might be exploited to replace the imidazole or indole moieties. As part of a programme to explore this possibility, we have previously reported on $\alpha$-amino acids $\mathbf{3}(n=0,2,3)$ carrying heterocycles in their side chains, including pyridine and isoxazole derivatives but in particular the pyrimidine and purine nucleobases tethered to the backbone with varying length carbon chains. ${ }^{1,2}$ These are potential $\alpha$ PNA monomers, ${ }^{3,4}$ amongst many other possible applications, and are analogues of natural products such as discadenine. ${ }^{5}$ They are obtained by stereoselective conjugate radical addition to an optically active methylene oxazolidinone derived from $S$-methylcysteine, ${ }^{1}$ or by stereoselective C-alkylation of an ephedrine-based glycinamide. ${ }^{2}$ In pursuit of further variation in the heterocycle-backbone tether and the backbone-to-heterocycle linkage protocol, and to demonstrate diversity in the heterocycle, we report now the preparation of 11 novel heterocyclic amino acids 4 ( $\mathrm{n}=1,2,3)$ with sulfur-containing tethers, obtained by elaboration of cysteine (Figure 1). ${ }^{6,7}$ The strategy adopted (Scheme 1) was to alkylate the side chain sulfur atom of a cysteine derivative with haloalkyl heterocycles; the heterocycles employed were nucleobases (uracil, thymine, adenine) and the simpler analogues indole, benzimidazole, benzotriazole and purine. This $\mathrm{C}-\mathrm{S}$ bond formation is distinct from those employed by others, and extends the 
portfolio of heterocycles beyond the nucleobases. ${ }^{3,6,7}$ The product amino acids are possible components in novel non-natural amino acid sequences with potential biological properties.

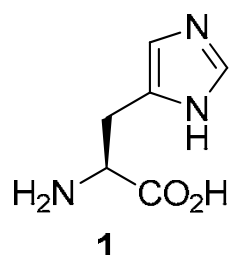

1

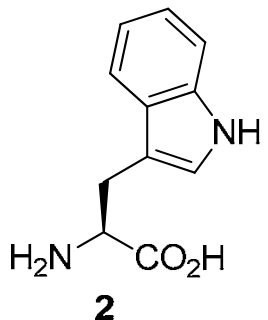

2

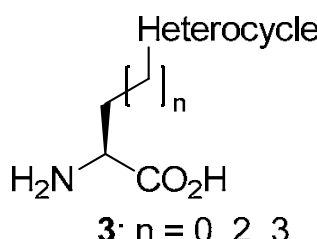

3; $n=0,2,3$

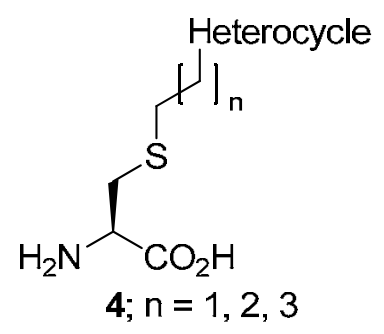

Figure 1. Natural and synthetic heterocyclic amino acids.
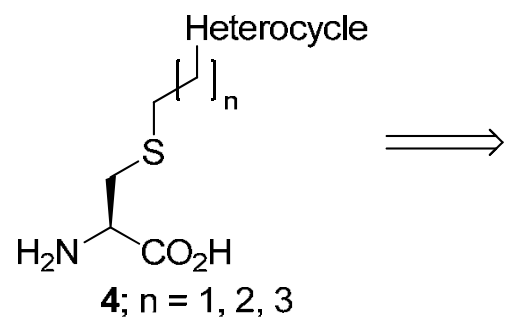

$\oplus$
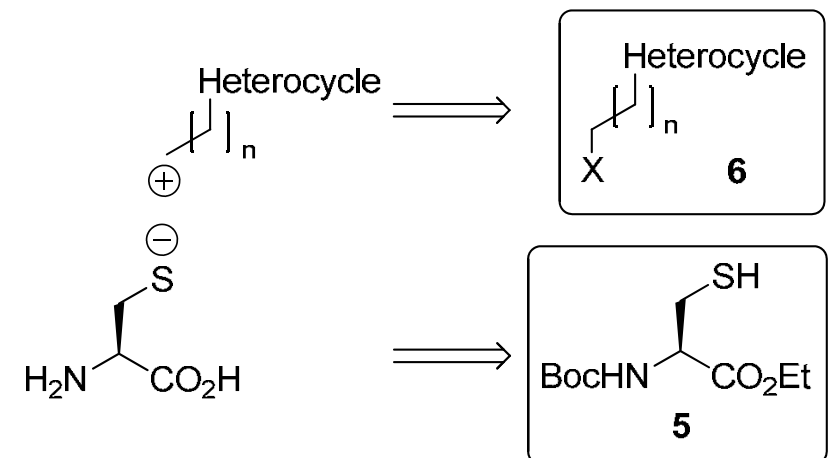

Scheme 1. Synthetic strategy for S-linked heterocyclic amino acids.

\section{Results and Discussion}

The scaffold used for the side chain elaboration was $N$-tert-butoxycarbonyl- $(R)$-cysteine ethyl ester 5, easily prepared from commercial $(R)$-cysteine ethyl ester hydrochloride $\left(\mathrm{Boc}_{2} \mathrm{O}, i\right.$ $\left.\mathrm{Pr}_{2} \mathrm{NEt}, \mathrm{CH}_{2} \mathrm{Cl}_{2}, 2 \mathrm{~h} ; 98 \%\right)$. Relevant electrophiles, the $\omega$-iodoalkyl heterocycles $6(\mathrm{n}=1,2,3)$, were prepared by two general methods (Scheme 2): (i) Method A, Mitsunobu coupling (i$\mathrm{PrO}_{2} \mathrm{CN}=\mathrm{NCO}_{2} \mathrm{i}$-Pr (DIAD), $\mathrm{Ph}_{3} \mathrm{P}$ ) of $\omega$-bromoalcohols 7 (2-bromoethanol, 3-bromopropan-1-ol, 4-bromobutan-1-ol) with NH-heterocycles, followed by Finkelstein halide exchange with iodide ion (NaI, $\mathrm{Me}_{2} \mathrm{CO}$ reflux); ${ }^{1}$ or (ii) Method $\mathrm{B}$, phase-transfer $\mathrm{N}$-alkylation $\left(\mathrm{Bu}_{4} \mathrm{NI}, \mathrm{KOH}, \mathrm{K}_{2} \mathrm{CO}_{3}\right)$ of the heterocycle with an $\alpha, \omega$-dihalide $\mathbf{8}$ (1,2-dichloroethane, 1,3-dichloropropane, 1,4dichlorobutane) used as solvent, again followed by halide exchange to generate the $\mathrm{N}-(\omega$ iodoalkyl) derivative. In this way, a range of $N$-iodoethyl derivatives $\mathbf{9 a - h}$ were prepared from 3benzoyluracil, 3-benzoylthymine, indole, benzimidazole, benzotriazole, $N^{6}$-(tertbutoxycarbonyl)adenine and purine as shown in Figure 2 and Table 1 . In the case of benzotriazole, both $\mathrm{N}-1$ and $\mathrm{N}-2$ bromoethyl derivatives were formed using method $\mathrm{A}$, and could be separated by column chromatography, whereas method B produced only the $\mathrm{N}-1$ derivative. 
In the same manner, $N$-iodopropyl derivatives 10e,f and $N$-iodobutyl derivatives 11e,f were also prepared from benzotriazole. Method A provided only the N-1 bromopropyl variant (although this was not taken further), but using method $\mathrm{B}$, both the $\mathrm{N}-1$ and $\mathrm{N}-2$ chloropropyl and chlorobutyl compounds were formed and were separated by column chromatography (Figure 1, Table 1). $N$-(3-Iodopropyl)indole 10c could not be isolated pure from the corresponding chloroalkyl compound, and the $\mathrm{N}$-(4-chlorobutyl) derivative could also not be purified from method B. When $N$-(3-chloropropyl)- or $N$-(3-bromopropyl)benzimidazole were subjected to our standard Finkelstein conditions with sodium iodide, the corresponding iodoalkylbenzimidazole 10d was not observed but a very polar material was isolated, provisionally assigned on the basis of MS and ${ }^{1} \mathrm{H}$ NMR spectroscopic evidence as the cyclic double quaternary salt 12a (Figure 3). ${ }^{8,9}$ Likewise, when formation of $\mathrm{N}$-(4-chlorobutyl)benzimidazole was attempted according to method B, a polar product similarly assigned as the cyclic double salt $\mathbf{1 2 b}$ was obtained.

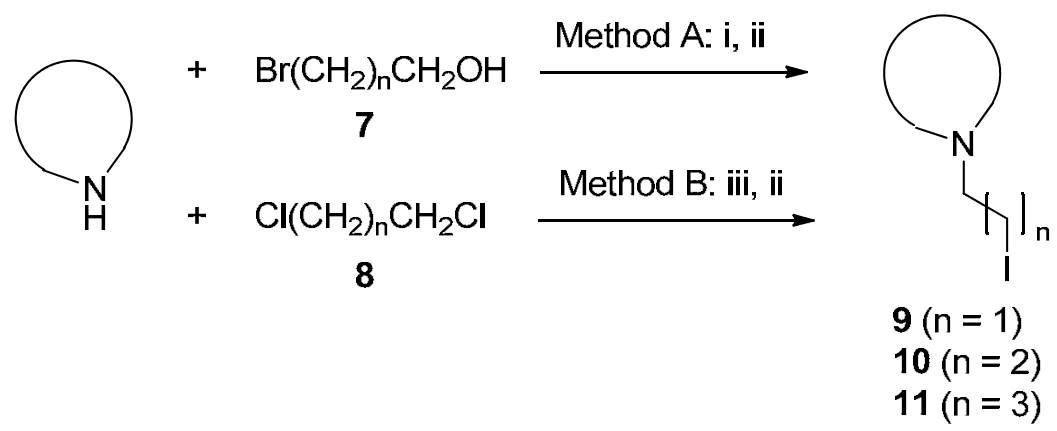

Scheme 2. Haloalkylation of NH-heterocycles. Reagents: i, DIAD, $\mathrm{Ph}_{3} \mathrm{P}$; ii, $\mathrm{NaI}, \mathrm{Me}_{2} \mathrm{CO}$ reflux; iii, $\mathrm{Bu}_{4} \mathrm{NI}, \mathrm{KOH}, \mathrm{K}_{2} \mathrm{CO}_{3}$.<smiles>O=C(c1ccccc1)n1c(=O)ccn(CCI)c1=O</smiles>

9a<smiles></smiles>

$9 \mathbf{e} ; n=1$

10e; $n=2$ $11 \mathrm{e} ; n=3$<smiles>Cc1cn(CCI)c(=O)n(C(=O)c2ccccc2)c1=O</smiles>

$9 b$<smiles>IC(I)Cn1nc2ccccc2n1</smiles>

9f; $n=1$ 10f; $n=2$ 11f; $n=3$<smiles>IC(I)CCn1ccc2ccccc21</smiles>

9c; $n=1$<smiles>CC(C)(C)Nc1ncnc2c1ncn2CCI</smiles>

9g<smiles>ICCn1cnc2ccccc21</smiles>

9d; $n=1$<smiles>ICCn1cnc2cncnc21</smiles>

9h

Figure 2. $\omega$-Iodoalkyl heterocycles 9-11 (9: $n=1 ; 10: n=2 ; 11: n=3)$ prepared according to Scheme 2. 
Table 1. Preparation of $\omega$-iodoalkyl heterocycles 9-11

\begin{tabular}{cccc}
\hline Product & Method & $\begin{array}{c}\text { Yield \% } \\
\text { (step 1) }\end{array}$ & Yield \% (halide exchange) \\
\hline 9a & A & $95 \%$ & $36 \%$ \\
9b & A & $56 \%$ & $54 \%$ \\
9c & B & $81 \%$ & $64 \%$ \\
9d & A & $98 \%$ & $75 \%$ \\
& A* & $80 \%$ & $72 \%$ \\
& B & $54 \%$ & $72 \%$ \\
9e & B & $66 \%$ & $94 \%$ \\
& A & $27 \%$ & $95 \%$ \\
9f & A & $56 \%$ & $97 \%$ \\
9g & A & $37 \%$ & $51 \%$ \\
& B & $59 \%$ & $64 \%$ \\
9h & A & $73 \%$ & $79 \%$ \\
& B & $59 \%$ & $64 \%$ \\
10c & B & $61 \%$ & $\dagger$ \\
10d & A & $78 \%$ & $\ddagger$ \\
& B & $62 \%$ & 7 \\
10e & A & $78 \%$ & - \\
& B & $52 \%$ & $77 \%$ \\
10f & B & $24 \%$ & $65 \%$ \\
11d & B & $\S$ & \\
11e & B & $24 \%$ & $70 \%$ \\
\hline
\end{tabular}

*using 2-choroethanol rather than 2-bromoethanol; $\dagger$ : impure product obtained; $\ddagger$ : product assigned as $\mathbf{1 2 a}$; $\S$ : product assigned as $\mathbf{1 2 b}$.

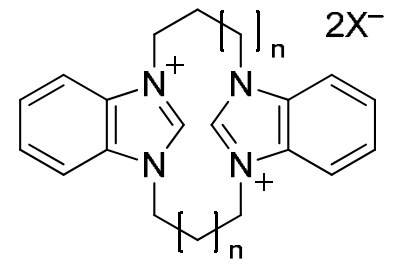

$$
\begin{aligned}
& 12 a ; n=1, X=I \\
& 12 b ; n=2, X=C l
\end{aligned}
$$

Figure 3. Products of benzimidazole haloalkylation.

With these electrophiles 9-11 in hand, we proceeded to successfully employ them in Salkylation of the protected cysteine $\mathbf{5}$ under basic conditions (Scheme 3). After a number of investigations, our preferred protocol was determined to be treatment of $\mathrm{N}$-protected cysteine 
ester 5 in dry THF under an atmosphere of nitrogen, with sodium hydride $\left(2.5 \mathrm{~mol}\right.$ eq) at $0{ }^{\circ} \mathrm{C}$ and the mixture allowed to warm to $20^{\circ} \mathrm{C}$ over 5-20 min, followed by addition of the iodoalkyl heterocycle (4 mol eq). If the halide was added directly after the base, alkylation yields were lower and elimination product was observed; an optimum delay in each case was determined by experiment. After stirring the alkylation reaction mixture under reflux for an appropriate period, normally $16 \mathrm{~h}$, the novel S-linked protected amino acids were isolated by conventional methods. In this way, 11 non-proteinogenic amino acid derivatives 13a-k were prepared in the yields shown in Figure 4; compound $\mathbf{1 3 g}$ was not fully characterised. Purine derivative $\mathbf{9 h}$ did not afford significant alkylation product under our conditions. The optical purity of the new residues remains to be determined.

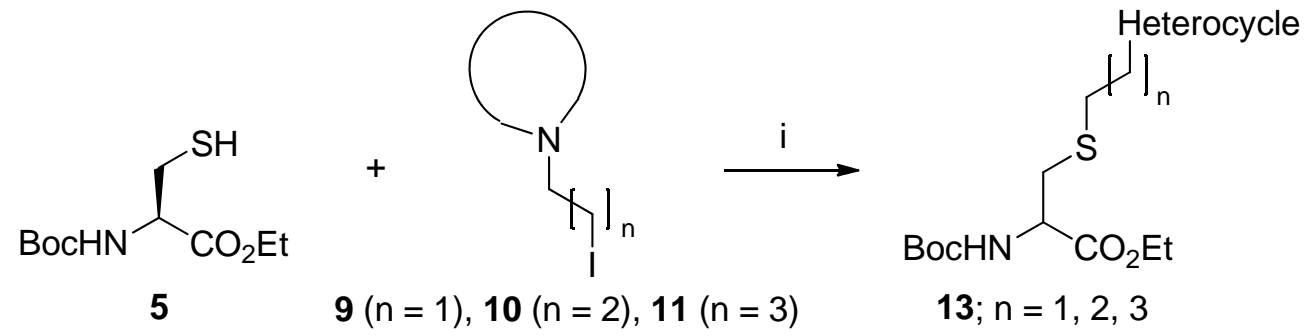

Scheme 3. S-alkylation of protected cysteine 5. Reagents: i, 5 with NaH, THF, 0 to $20{ }^{\circ} \mathrm{C}, 5-20$ min, then halide $\mathbf{9 , 1 0}$ or 11.
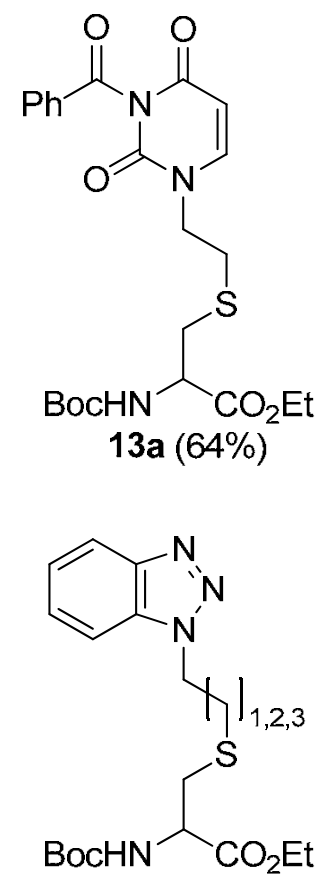

13e, $\mathbf{h}, \mathbf{j}(n=1,2,3$;

$75,59,39 \%$ respectively)<smiles>CCOC(=O)C(CSCCn1cc(C)c(=O)n(C(=O)c2ccccc2)c1=O)NC(=O)OC(C)(C)C</smiles>

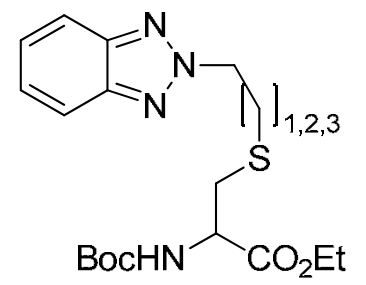

13f, $\mathbf{i}, \mathbf{k}(\mathrm{n}=1,2,3$ $74,68,61 \%$ respectively)
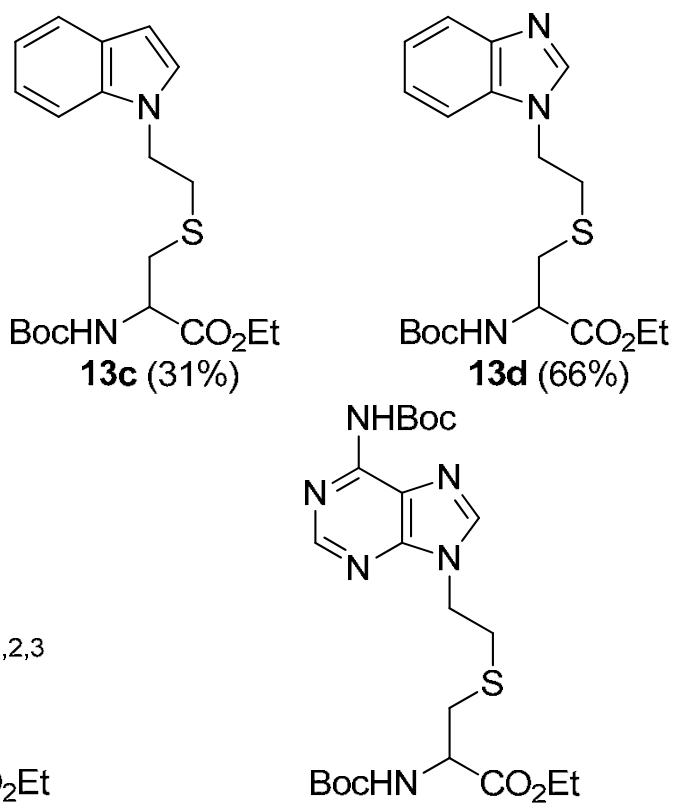

$13 \mathbf{g}(41 \%$; not fully characterised)

Figure 4. S-Linked heterocyclic amino acids prepared according to Scheme 3. 
To demonstrate the potential for application of the new amino acids in organic synthesis, the benzimidazole-carrying derivative 13d was orthogonally deprotected (Scheme 4) at either its Cor N-terminus. Thus, basic hydrolysis (1M LiOH aq, THF-MeOH, $20{ }^{\circ} \mathrm{C}, 8 \mathrm{~h}$ ) afforded the $\mathrm{N}$ protected acid 14 (97\%). Alternatively, acid treatment (TFA, $\mathrm{CH}_{2} \mathrm{Cl}_{2}, 20{ }^{\circ} \mathrm{C}, 2 \mathrm{~h}$ ) and subsequent neutralization ( $i-\mathrm{Pr}_{2} \mathrm{NEt}$ ) led to the free amine 15 (86\%).
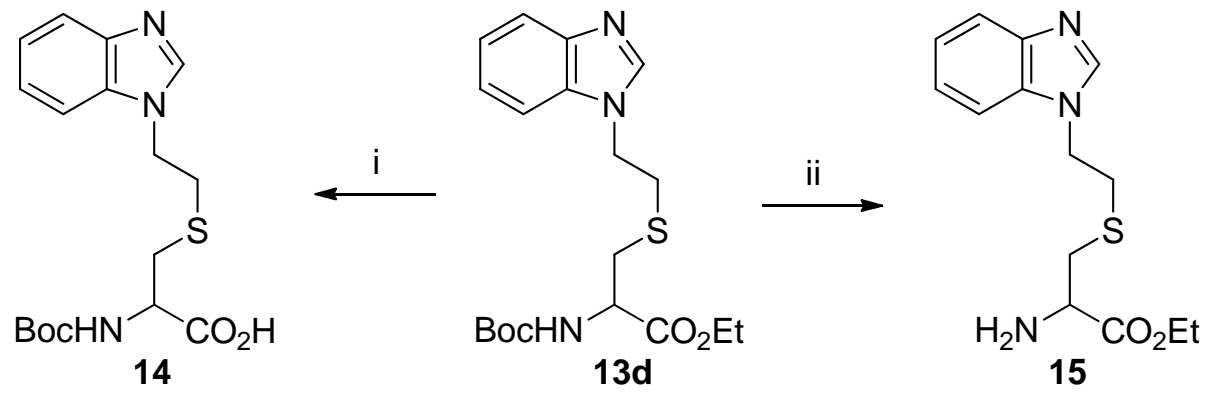

Scheme 4. Deprotection of S-linked amino acid derivative 13d. Reagents: i, LiOH aq, THF$\mathrm{MeOH}, 2{ }^{\circ} \mathrm{C}, 8 \mathrm{~h}$; ii, TFA, $\mathrm{CH}_{2} \mathrm{Cl}_{2}, 2{ }^{\circ} \mathrm{C}, 2 \mathrm{~h}$, then $i-\mathrm{Pr}_{2} \mathrm{Net}$.

A similar approach using $N$-benzyloxycarbonyl-(S)-serine methyl ester to give O-linked heterocyclic amino acids, was unsuccessful, as base treatment led to polymerisation rather than O-alkylation. Using a more hindered tert-butyl ester afforded no improvement. Alkylation using the free acid $N$-benzyloxycarbonyl-(S)-serine showed possible success that awaits further study. ${ }^{10}$ Investigations with 2,3-diaminopropionic acid and 2,4-diaminobutyric acid were curtailed as a suitable protected amino acid alkylation substrate could not be easily obtained. On the other hand, we have indications that our approach will be successful using a protected homocysteine scaffold. ${ }^{11}$

\section{Conclusions}

We have successfully prepared 11 novel heterocyclic $\alpha$-amino acids by S-alkylation of an $\mathrm{N}$ protected cysteine ester, and demonstrated the orthogonal deprotection that will enable their application in organic synthesis.

\section{Experimental Section}

General. Flash column chromatography was performed using silica gel 60 (40-63 $\mu$, 230-400 mesh, $60 \mathrm{~A}$ ), and thin layer chromatography (TLC) performed on Merck $0.2 \mathrm{~mm}$ silica $60 \mathrm{~F}_{254}$ coated UV active aluminium sheets. Melting Points were measured on a Stuart Scientific Bibby SMP3 Melting Point Apparatus and IR spectra were recorded on a Perkin-Elmer Paragon 1000 
FTIR spectrometer for neat samples on $\mathrm{NaCl}$ discs $\left(\mathrm{CH}_{2} \mathrm{Cl}_{2}\right.$ used as a 'transport' solvent where necessary). ${ }^{1} \mathrm{H}$ NMR spectra were recorded at $400 \mathrm{MHz}$ on a Bruker DPX-400 instrument or at $250 \mathrm{MHz}$ on a Bruker AC-250 instrument; ${ }^{13} \mathrm{C}$ NMR spectra were recorded at $100 \mathrm{MHz}$ on the Bruker DPX-400 instrument. Chemical shifts are quoted as $\delta$ ppm with TMS as internal standard, and the coupling constants $J$ in Hz. Signals are described as s (singlet), d (doublet), t (triplet), q (quartet), m (multiplet), br (broad). Mass spectra were recorded using either EI or FAB ionisation, on a JEOL SX102 spectrometer at Loughborough University or by the ESPRC National Mass Spectrometry Service Centre at Swansea University. Liquid ChromatographyMass Spectrometry was performed using a Waters 600 Controller instrument, a Waters Symmetry C8 $3.5 \mu \mathrm{m} 4.6$ x $50 \mathrm{~nm}$ column and a Waters 996 photodiode array detector attached to a Micromass Platform mass spectrometer using electrospray ionization. Elemental Analyses for $\mathrm{C}, \mathrm{H}$ and $\mathrm{N}$ were obtained using a Perkin-Elmer CHN-2400 Elemental Analyzer. 2Bromoethanol, 2-chloroethanol, 3-bromopropan-1-ol, 4-bromobutan-1-ol, 1,2-dichloroethane, 1,3-dichloropropane, 1,4-dichlorobutane, uracil, thymine, indole, benzimidazole, benzotriazole, adenine, purine and $(R)$-cysteine ethyl ester were purchased from either Aldrich, Lancaster or Avocado and were used without purification unless otherwise stated. 3-Benzoyluracil and 3benzoylthymine were prepared according to the method of Reese et al. ${ }^{12}$ Solvents were dried where necessary, as follows: THF was pre-dried in the presence of solid $\mathrm{K}_{2} \mathrm{CO}_{3}$ then distilled from sodium metal and benzophenone under a positive atmosphere of nitrogen; EtOAc was distilled over calcium chloride; $\mathrm{CH}_{2} \mathrm{Cl}_{2}$ was distilled over anhydrous calcium hydride; and 1,4dioxane was shaken over potassium hydroxide pellets. Characterization data are given for pure compounds.

\section{Method A. General procedure for heterocycle $\omega$-bromoalkylation}

$\mathrm{NH}$-Heterocycle ( 1 mol equiv), triphenylphosphine (1.2 mol equiv) and the $\omega$-bromoalcohol (1.2 mol equiv) were suspended in dry dioxane ( $50 \mathrm{~mL}$ per mmol of heterocycle) at $5{ }^{\circ} \mathrm{C}$, to which was added DIAD (1.2 mol equiv) dropwise over $3 \mathrm{~h}$. The mixture was stirred under an atmosphere of nitrogen at $20^{\circ} \mathrm{C}$ to give a clear solution. The solvent was removed under reduced pressure and the residue purified by flash chromatography to give the pure title compound as a yellow oil, which was in some cases recrystallised to give the product.

\section{Method B. General procedure for heterocycle $\omega$-chloroalkylation}

Heterocycle ( 1 mol equiv.) was added to a mixture of $\alpha, \omega$-dichloroalkane, which acted as both the solvent and the reagent, tetrabutylammonium iodide $(5 \% \mathrm{w} / \mathrm{w})$ as the phase-transfer catalyst, $\mathrm{KOH}$ (6 mol equiv.) and $\mathrm{K}_{2} \mathrm{CO}_{3}\left(2.5 \mathrm{~mol}\right.$ equiv.). The reaction was stirred at $20^{\circ} \mathrm{C}$ for $4 \mathrm{~h}$. The inorganic material was filtered off and the organic portion washed with water ( $5 \mathrm{~mL}$ per $\mathrm{mmol}$ of heterocycle), dried $\left(\mathrm{MgSO}_{4}\right)$, filtered and the solvent removed under reduced pressure. The mixture was then purified by flash column chromatography to isolated the pure chloroalkyl compound. 


\section{General procedure $C$. Halide exchange to afford $\omega$-iodoalkyl heterocycles (9-11)}

$\mathrm{N}$-( $\omega$-Haloalkyl) heterocycle ( $1 \mathrm{~mol}$ equiv) and dry sodium iodide (5 mol equiv) were heated in dry acetone (50 $\mathrm{mL}$ per mmol of heterocycle) at reflux overnight in the dark under a nitrogen atmosphere. After cooling, the acetone was removed under reduced pressure and the residue taken up in EtOAc : water $(1: 1 \mathrm{v} / \mathrm{v})$, the organic layer was separated and the aqueous layer was extracted twice more with EtOAc. The combined organic layers were washed twice with sodium thiosulfate solution $(2 \% \mathrm{w} / \mathrm{v})$, dried $\left(\mathrm{MgSO}_{4}\right)$, filtered and the solvent was removed under reduced pressure to yield the pure iodoalkyl compound.

3-Benzoyl-1-(2-bromoethyl)uracil. Prepared according to method A as previously reported, using 3-benzoyluracil (5.00 g, $23.04 \mathrm{mmol})$, triphenylphosphine (7.24 g, $27.63 \mathrm{mmol}$ ) and 2bromoethanol $(1.96 \mathrm{~mL}, 3.45 \mathrm{~g}, 27.62 \mathrm{mmol})$ in dry dioxane $(100 \mathrm{~mL})$ at $5{ }^{\circ} \mathrm{C}$ with DIAD (5.43 $\left.\mathrm{cm}^{3}, 5.58 \mathrm{~g}, 27.61 \mathrm{mmol}\right)$ to give the title compound as a white solid (7.07 g, 95\%), m.p. 177$179{ }^{\circ} \mathrm{C}$ (lit., ${ }^{1}$ m.p. $\left.183-184{ }^{\circ} \mathrm{C}\right)$, IR ( $\left.v_{\max }, \mathrm{NaCl} / \mathrm{cm}^{-1}\right)$ : 2920, 2850, 1747, 1704, 1662, 1437, 1346 , 1241. ${ }^{1} \mathrm{H}$ NMR (400 MHz, $\mathrm{CDCl}_{3}$ ), $\delta_{\mathrm{H}} 3.69,4.16$ (each $2 \mathrm{H}, \mathrm{t}, J=5.6 \mathrm{~Hz}, \mathrm{CH}_{2} \mathrm{CH}_{2}$ ), $5.83(1 \mathrm{H}, \mathrm{d}$, $J=8.0 \mathrm{~Hz}, 5-\mathrm{CH}$ ), 7.33, 7.51 (each 2H, t, $J=8.0 \mathrm{~Hz}, \mathrm{Ar}-\mathrm{H}$ ), 7.67 (1H, d, $J=8.0 \mathrm{~Hz}, \mathrm{Ar}-\mathrm{H}$ ), 7.93 $(1 \mathrm{H}, \mathrm{d}, J=8.0 \mathrm{~Hz}, 6-\mathrm{CH}) .{ }^{13} \mathrm{C} \mathrm{NMR}\left(100 \mathrm{MHz}, \mathrm{CDCl}_{3}\right), \delta_{\mathrm{C}} 29.4\left(\mathrm{CH}_{2} \mathrm{Br}\right), 51.3\left(\mathrm{CH}_{2} \mathrm{~N}\right), 101.9$ (5-CH), 129.3, 130.5 (2 x Ar-CH), 132.1 (Ar-C), 135.2 (Ar-CH), 144.8 (6-CH), 149.7, 159.9, 168.1 (3 x C=O). MS, $m / z=324 / 322\left(\mathrm{M}^{+}\right)$, 296, 294, 277, 254, 252, 215, 188, 105.

3-Benzoyl-1-(2-iodoethyl)uracil (9a). Prepared according to general procedure $\mathrm{C}$ as previously reported, using 3-benzoyl-1-(2-bromoethyl)uracil (5.00 g, $15.48 \mathrm{mmol}$ ) and dry NaI (11.71 g, $78.07 \mathrm{mmol})$ in dry acetone $(100 \mathrm{~mL})$ to yield the title compound as a pale yellow solid (2.11 $\mathrm{g}$, 36\%), m.p. $185-18{ }^{\circ} \mathrm{C}$ (lit., ${ }^{1}$ m.p. $\left.190-19{ }^{\circ} \mathrm{C}\right)$, IR $\left(v_{\max }, \mathrm{NaCl} / \mathrm{cm}^{-1}\right)$ 2920, 2850, 1747, 1705, 1664, 1436, 1254. ${ }^{1} \mathrm{H}$ NMR (400 MHz, $\mathrm{CDCl}_{3}$ ), $\delta_{\mathrm{H}} 3.48,4.11$ (each $2 \mathrm{H}, \mathrm{t}, J=6.4 \mathrm{~Hz}, \mathrm{CH}_{2} \mathrm{CH}_{2}$ ), $5.84(1 \mathrm{H}, \mathrm{d}, J=8.0 \mathrm{~Hz}, 5-\mathrm{CH}), 7.28,7.51$ (each $2 \mathrm{H}, \mathrm{t}, J=8.0 \mathrm{~Hz}, \mathrm{Ar}-\mathrm{H}), 7.66(1 \mathrm{H}, \mathrm{d}, J=8.0$ $\mathrm{Hz}, 6-\mathrm{CH}), 7.93(1 \mathrm{H}, \mathrm{d}, J=8.0 \mathrm{~Hz}, \mathrm{ArH}) .{ }^{13} \mathrm{C} \mathrm{NMR}\left(100 \mathrm{MHz}, \mathrm{CDCl}_{3}\right), \delta_{\mathrm{C}} 22.9\left(\mathrm{CH}_{2} \mathrm{I}\right), 51.8$ $\left(\mathrm{CH}_{2} \mathrm{~N}\right), 102.0$ (5-CH), 129.2, 130.5 (2 x Ar-CH), 133.3 (Ar-C), 135.9 (Ar-CH), 144.2 (6-CH), 149.1, 162.5, 171.1 (3 x C=O). MS, $m / z=370\left(\mathrm{M}^{+}\right)$, 342, 277, 215, 188, 155, 105.

3-Benzoyl-1-(2-bromoethyl)thymine. Prepared according to method A as previously reported, using 3-benzoylthymine (5.00 g, $21.65 \mathrm{mmol})$, triphenylphosphine (6.84 g, $26.11 \mathrm{mmol})$ and 2bromoethanol $(1.85 \mathrm{~mL}, 3.36 \mathrm{~g}, 26.08 \mathrm{mmol})$ in dry dioxane $(100 \mathrm{~mL})$ at $5{ }^{\circ} \mathrm{C}$ with DIAD $(5.13$ $\mathrm{cm}^{3}, 5.27 \mathrm{~g}, 26.08 \mathrm{mmol}$ ) to give the title compound as a white solid (7.32 g, 56\%), m.p. 166$167^{\circ} \mathrm{C}$ (lit., ${ }^{1}$ m.p. $\left.183-184^{\circ} \mathrm{C}\right)$, IR $\left(v_{\max }, \mathrm{NaCl} / \mathrm{cm}^{-1}\right)$ 2898, 2342, 1746, 1699, 1662, 1462, 1414 , 1429, 1376. ${ }^{1} \mathrm{H}$ NMR (400 MHz, $\mathrm{CDCl}_{3}$ ), $\delta_{\mathrm{H}} 1.98\left(3 \mathrm{H}, \mathrm{s}, \mathrm{CH}_{3}\right.$ ), 3.44, 4.07 (each $2 \mathrm{H}, \mathrm{t}, J=6.6$ $\left.\mathrm{Hz}, \mathrm{CH}_{2} \mathrm{CH}_{2}\right), 7.13(1 \mathrm{H}, \mathrm{d}, J=1.2 \mathrm{~Hz}, 6-\mathrm{CH}), 7.50(2 \mathrm{H}, \mathrm{t}, J=7.4 \mathrm{~Hz}, \mathrm{Ar}-\mathrm{H}), 7.65$ (1H, t, $J=7.4$ $\mathrm{Hz}, \mathrm{Ar}-\mathrm{H}), 7.93(2 \mathrm{H}, \mathrm{d}, J=7.4 \mathrm{~Hz}, \mathrm{Ar}-\mathrm{H}) .{ }^{13} \mathrm{C} \mathrm{NMR}\left(100 \mathrm{MHz}, \mathrm{CDCl}_{3}\right), \delta_{\mathrm{C}} 11.6\left(\mathrm{CH}_{3}\right), 36.7$ $\left(\mathrm{CH}_{2} \mathrm{Br}\right), 50.4\left(\mathrm{CH}_{2} \mathrm{~N}\right), 109.7$ (5-C), 128.3, 129.6 (2 x Ar-CH), 130.6 (Ar-C), 134.2 (Ar-CH), 139.3 (6-CH), 148.7, 162.1, 167.8 (3 x C=O). MS, $m / z=338 / 336\left(\mathrm{M}^{+}\right)$, 310, 308, 277, 254, 252, 230, 188, 105. HRMS: Calcd for $\mathrm{C}_{14} \mathrm{H}_{13}{ }^{79} \mathrm{BrN}_{2} \mathrm{O}_{3}: \mathrm{M}^{+}$336.0110; found: $\mathrm{M}^{+}$336.0112.

3-Benzoyl-1-(2-iodoethyl)thymine (9b). Prepared according to general procedure $\mathrm{C}$ as previously reported, using 3-benzoyl-1-(2-bromoethyl)thymine (5.00 g, $14.84 \mathrm{mmol}$ ) and dry 
$\mathrm{NaI}(11.11 \mathrm{~g}, 74.07 \mathrm{mmol})$ in dry acetone $(100 \mathrm{~mL})$ to yield the title compound as a pale yellow solid (3.11 g, 54\%), m.p. 145-147 ${ }^{\circ} \mathrm{C}$ (lit., ${ }^{1}$ m.p. 138-139 $\left.{ }^{\circ} \mathrm{C}\right)$, IR ( $v_{\max }\left(\mathrm{NaCl} / \mathrm{cm}^{-1}\right)$ 2954, 1743, 1702, 1640, 1451, 1411, 1363. ${ }^{1} \mathrm{H}$ NMR (400 MHz, $\left.\mathrm{CDCl}_{3}\right), \delta_{\mathrm{H}} 2.04\left(3 \mathrm{H}, \mathrm{s}, \mathrm{CH}_{3}\right), 3.57,4.33$ (each 2H, t, $J=6.8 \mathrm{~Hz}, \mathrm{CH}_{2} \mathrm{CH}_{2}$ ), $7.47(2 \mathrm{H}, \mathrm{t}, J=7.4 \mathrm{~Hz}, \mathrm{Ar}-\mathrm{H}), 7.62(1 \mathrm{H}, \mathrm{t}, J=7.4 \mathrm{~Hz}, \mathrm{Ar}-\mathrm{H})$, $7.66(1 \mathrm{H}, \mathrm{d}, J=2.6 \mathrm{~Hz}, 6-\mathrm{CH}), 7.75(2 \mathrm{H}, \mathrm{d}, J=7.4 \mathrm{~Hz}, \mathrm{Ar}-\mathrm{H}) .{ }^{13} \mathrm{C}$ NMR $\left(100 \mathrm{MHz}, \mathrm{CDCl}_{3}\right), \delta_{\mathrm{C}}$ $13.2\left(\mathrm{CH}_{3}\right), 29.4\left(\mathrm{CH}_{2} \mathrm{I}\right), 42.0\left(\mathrm{CH}_{2} \mathrm{~N}\right), 112.3(5-\mathrm{C}), 128.6,129.3$ (2 x Ar-CH), 132.8 (Ar-C), 134.0 (Ar-CH), 140.7 (6-CH), 149.9, 162.9, 169.7 (3 x C=O). MS, $m / z=384\left(\mathrm{M}^{+}\right), 356,277$, 215, 230, 155, 105.

$\mathrm{N}$-(2-Chloroethyl)indole. Prepared according to method $\mathrm{B}$, using indole (2.00 g, $17.09 \mathrm{mmol})$, 1,2-dichloroethane (150 mL), tetrabutylammonium iodide (0.10 g, 5\% w/w), KOH (5.75 g, 0.10 mol) and $\mathrm{K}_{2} \mathrm{CO}_{3}(5.90 \mathrm{~g}, 0.04 \mathrm{~mol})$. After stirring for $24 \mathrm{~h}$, work up gave the title compound as a brown oil (2.46 g, 81\%), IR ( $\left.v_{\max }, \mathrm{NaCl} / \mathrm{cm}^{-1}\right)$ 3050, 2959, 1614, 1454, 1334, 1093. ${ }^{1} \mathrm{H} \mathrm{NMR}$ (400 MHz, $\mathrm{CDCl}_{3}$ ), $\delta_{\mathrm{H}} 3.84,4.48$ (each $2 \mathrm{H}, \mathrm{t}, J=6.6 \mathrm{~Hz}, \mathrm{CH}_{2} \mathrm{CH}_{2}$ ), 6.61 (1H, m, indole-CH), 7.18-7.28 (3H, m, 2 x Ar-H, indole-CH), 7.43, 7.71 (each $1 \mathrm{H}, \mathrm{d}, J=7.9 \mathrm{~Hz}, \mathrm{Ar}-\mathrm{H}) .{ }^{13} \mathrm{C}$ NMR $\left(100 \mathrm{MHz}, \mathrm{CDCl}_{3}\right), \delta_{\mathrm{C}} 42.5\left(\mathrm{CH}_{2} \mathrm{Cl}\right), 48.0\left(\mathrm{CH}_{2} \mathrm{~N}\right), 102.0,111.0$ (2 x indole-CH), 120.0, 121.9, 124.2 (3 x Ar-CH), 127.9 (Ar C), 128.8 (Ar-CH), 135.8 (Ar-C). MS, $m / z=180\left(\mathrm{MH}^{+}\right), 144,116$, 90. Anal. Calcd for $\mathrm{C}_{10} \mathrm{H}_{10} \mathrm{ClN}$ : C, 66.86; H, 5.61; N, 7.80\%, Found: C, 66.84; H, 5.91; N, $7.63 \%$.

$\mathrm{N}$-(2-Iodoethyl)indole (9c). Prepared according to general procedure C, using $\mathrm{N}$-(2chloroethyl)indole $(1.40 \mathrm{~g}, 7.80 \mathrm{mmol})$ and dry $\mathrm{NaI}(5.84 \mathrm{~g}, 38.93 \mathrm{mmol})$ in dry acetone (100 $\mathrm{mL}$ ). After work up this gave the title compound as a dark brown oil (1.36 g, 64\%), IR ( $v_{\max }$, $\mathrm{NaCl} / \mathrm{cm}^{-1}$ ) 3031, 2924, 1563, 1439, 1329, 1234, 1126. ${ }^{1} \mathrm{H}$ NMR (400 MHz, $\left.\mathrm{CDCl}_{3}\right), \delta_{\mathrm{H}} 3.44$, 4.55 (each 2H, t, $\left.J=7.7 \mathrm{~Hz}, \mathrm{CH}_{2} \mathrm{CH}_{2}\right), 6.60$ (1H, m, indole- $\left.\mathrm{CH}\right), 7.41-7.92(5 \mathrm{H}, \mathrm{m}, 4 \mathrm{x} \mathrm{Ar}-\mathrm{H}$, indole-CH). ${ }^{13} \mathrm{C}$ NMR $\left(100 \mathrm{MHz}, \mathrm{CDCl}_{3}\right), \delta_{\mathrm{C}} 44.3\left(\mathrm{CH}_{2} \mathrm{I}\right), 59.2\left(\mathrm{CH}_{2} \mathrm{~N}\right), 101.9$, $106.7(2 \mathrm{x}$ indole-CH), 109.7 (Ar-C), 119.2, 127.3, 127.8, 134.1 (4 x Ar-CH), 145.5 (Ar-C). MS, m/z = 272 $\left(\mathrm{MH}^{+}\right), 130,117,90$. Anal. Calcd for $\mathrm{C}_{10} \mathrm{H}_{10} \mathrm{IN}$ : C, 44.30; H, 3.72; N, 5.17\%, Found: C, 44.33; H, 3.90; N, 5.04\%.

$\boldsymbol{N}^{\mathbf{1}}$-(2-Bromoethyl)benzimidazole. Prepared according to method A, using benzimidazole (2.00 g, $16.95 \mathrm{mmol}$ ), triphenylphosphine (5.33 g, $20.34 \mathrm{mmol}$ ) and 2-bromoethanol (1.44 mL, $2.53 \mathrm{~g}$, $20.28 \mathrm{mmol})$ in dry dioxane $(100 \mathrm{~mL})$ at $5{ }^{\circ} \mathrm{C}$ with DIAD $(4.00 \mathrm{~mL}, 4.11 \mathrm{~g}, 20.34 \mathrm{mmol})$ to yield the title compound as colourless crystals (3.75 g, 98\%), m.p. $77-79{ }^{\circ} \mathrm{C}$, IR $\left(v_{\max }, \mathrm{NaCl} / \mathrm{cm}^{-1}\right)$ 2980, 2360, 1719, 1497, 1438, 1246. ${ }^{1} \mathrm{H}$ NMR (400 MHz, $\mathrm{CDCl}_{3}$ ), $\delta_{\mathrm{H}} 3.71,4.61$ (each $2 \mathrm{H}, \mathrm{t}, J=$ $6.0 \mathrm{~Hz}, \mathrm{CH}_{2} \mathrm{CH}_{2}$ ), 7.23-7.62 (4H, m, Ar-H), $7.90(1 \mathrm{H}, \mathrm{s}, 2-\mathrm{CH}) .{ }^{13} \mathrm{C} \mathrm{NMR}\left(100 \mathrm{MHz}, \mathrm{CDCl}_{3}\right), \delta_{\mathrm{C}}$ $39.2\left(\mathrm{CH}_{2} \mathrm{Br}\right), 45.1\left(\mathrm{CH}_{2} \mathrm{~N}\right), 109.2$ (2-CH), 119.3, 123.1, 129.0, 130.4 (4 x Ar-CH), 133.7, 144.9 ( 2 x ArC). MS, $m / z=226 / 224\left(M^{+}\right), 145,131,118,104,90$. HRMS: Calcd for $\mathrm{C}_{9} \mathrm{H}_{9}{ }^{79} \mathrm{BrN}_{2}: \mathrm{M}^{+}$ 223.9950; found: $\mathrm{M}^{+} 223.9950$.

$\boldsymbol{N}^{\mathbf{1}}$-(2-Chloroethyl)benzimidazole. Prepared according to method $\mathrm{B}$, using benzimidazole (1.00 g, $8.47 \mathrm{mmol})$ in 1,2-dichloroethane $(70 \mathrm{~mL})$ with tetrabutylammonium iodide $(0.05 \mathrm{~g}, 5 \% \mathrm{w} / \mathrm{w})$, $\mathrm{KOH}$ (2.85 g, $50.89 \mathrm{mmol}$ ) and $\mathrm{K}_{2} \mathrm{CO}_{3}$ (2.93 g, $21.23 \mathrm{mmol}$ ). After stirring for $4 \mathrm{~h}$, work up gave title compound (0.85 g, 54\%), m.p. $76-78{ }^{\circ} \mathrm{C}$ (lit., ${ }^{13}$ m.p. $\left.84-86{ }^{\circ} \mathrm{C}\right)$, IR $\left(v_{\max }, \mathrm{NaCl} / \mathrm{cm}^{-1}\right) 3056$, 
2960, 2359, 1615, 1494, 1437, 1257. ${ }^{1} \mathrm{H}$ NMR (400 MHz, $\mathrm{CDCl}_{3}$ ), $\delta_{\mathrm{H}} 3.76,4.42$ (each 2H, t, $J=$ 6.0 Hz, $\left.\mathrm{CH}_{2} \mathrm{CH}_{2}\right), 7.22-7.45$ (3H, m, Ar-H,), 7.59 (1H, m, Ar-H), $7.89(1 \mathrm{H}, \mathrm{s}, 2-\mathrm{CH}) .{ }^{13} \mathrm{C}$ NMR $\left(100 \mathrm{MHz}, \mathrm{CDCl}_{3}\right), \delta_{\mathrm{C}} 42.5\left(\mathrm{CH}_{2} \mathrm{Cl}\right), 47.0\left(\mathrm{CH}_{2} \mathrm{~N}\right), 109.6(2-\mathrm{CH}), 121.0,122.9,129.0,132.4(4 \mathrm{x}$ Ar-CH), 133.3, 143.7 ( 2 x Ar-C). MS, $m / z=180\left(\mathrm{M}^{+}\right)$, 144, 131, 117, 104, 90. HRMS: Calcd for $\mathrm{C}_{9} \mathrm{H}_{9}{ }^{35} \mathrm{ClN}_{2}$ : $\mathrm{M}^{+}$180.0454; found: $\mathrm{M}^{+}$180.0458. Anal. Calcd for $\mathrm{C}_{9} \mathrm{H}_{9} \mathrm{ClN}_{2}$ : C, 59.84; $\mathrm{H}$, 5.02; N, 15.51\%, Found: C, 60.28; H, 5.22; N, 15.98\%.

Also prepared by a modification to method A, using benzimidazole (1.00 g, $8.47 \mathrm{mmol})$, triphenylphosphine (2.66 g, $10.15 \mathrm{mmol})$ and 2-chloroethanol $(0.82 \mathrm{~g}, 10.19 \mathrm{mmol})$ in dry dioxane (20 mL) at $5{ }^{\circ} \mathrm{C}$ with DIAD (2.00 mL, $\left.2.05 \mathrm{~g}, 10.17 \mathrm{mmol}\right)$. After stirring for $16 \mathrm{~h}$, work up gave title compound $(1.23 \mathrm{~g}, 80 \%)$. Data as for material prepared using method $\mathrm{B}$.

$N^{1}$-(2-Iodoethyl)benzimidazole (9d). Prepared according to general procedure C, using $N^{1}$-(2chloroethyl)benzimidazole (2.00 g, $11.08 \mathrm{mmol})$ and dry NaI $(8.12 \mathrm{~g}, 54.13 \mathrm{mmol})$ in dry acetone $(100 \mathrm{~mL})$. Work up gave the title compound as a pale yellow solid (2.12 g, 72\%), IR $\left(v_{\max }\left(\mathrm{NaCl} / \mathrm{cm}^{-1}\right) 2980,1653,1506,1374,1240,1108 .{ }^{1} \mathrm{H}\right.$ NMR $\left(400 \mathrm{MHz}, \mathrm{CDCl}_{3}\right), \delta_{\mathrm{H}} 3.51$, 4.58 (each $2 \mathrm{H}, \mathrm{t}, J=7.2 \mathrm{~Hz}, \mathrm{CH}_{2} \mathrm{CH}_{2}$ ), 7.34-7.53 (3H, m, Ar-H,), $7.83(1 \mathrm{H}, \mathrm{m}, \mathrm{Ar}-\mathrm{H}), 7.99(1 \mathrm{H}$, s, 2-CH). ${ }^{13} \mathrm{C}$ NMR (100 MHz, $\left.\mathrm{CDCl}_{3}\right), \delta_{\mathrm{C}} 44.2\left(\mathrm{CH}_{2} \mathrm{I}\right), 53.1\left(\mathrm{CH}_{2} \mathrm{~N}\right), 109.9(2-\mathrm{CH}), 121.3$, 121.9, 124.9, 130.2 (4 x Ar-CH), 140.8, 146.4 (2 x Ar-C). MS, $m / z=272\left(\mathrm{M}^{+}\right), 144,131,118$, 104, 90. HRMS: Calcd for $\mathrm{C}_{9} \mathrm{H}_{9} \mathrm{IN}_{2}: \mathrm{M}^{+}$271.9812; found: $\mathrm{M}^{+} 271.9808$.

Also prepared according to general procedure C, but using $N^{1}$-(2-bromoethyl)benzimidazole $(2.00 \mathrm{~g}, 8.89 \mathrm{mmol})$ and dry NaI $(6.66 \mathrm{~g}, 44.40 \mathrm{mmol})$ in dry acetone $(100 \mathrm{~mL})$. Work up gave the title compound as a pale yellow solid (1.82 g, 75\%). Data as for material prepared using the 2-chloroethyl derivative.

$N^{1}$ - and $N^{2}$-(2-Bromoethyl)benzotriazole. Prepared according to method A, using benzotriazole ( $2.00 \mathrm{~g}, 16.81 \mathrm{mmol})$, triphenylphosphine $(5.28 \mathrm{~g}, 20.15 \mathrm{mmol})$ and 2-bromoethanol (1.43 mL, $2.52 \mathrm{~g}, 20.16 \mathrm{mmol})$ in dry dioxane (100 mL) with DIAD (3.97 mL, $4.08 \mathrm{~g}, 20.18 \mathrm{mmol})$. After workup and column chromatography this yielded the title compounds. $N^{1}$-(2Bromoethyl)benzotriazole: yellow crystals (1.03 g, 27\%), m.p. $107^{\circ} \mathrm{C}$ (lit., ${ }^{14}$ m.p. $119-120{ }^{\circ} \mathrm{C}$ ), IR $\left(v_{\max }, \mathrm{NaCl} / \mathrm{cm}^{-1}\right)$ 2929, 1650, 1444, 1329, 1249. ${ }^{1} \mathrm{H}$ NMR (400 MHz, $\left.\mathrm{CDCl}_{3}\right), \delta_{\mathrm{H}} 3.88,5.03$ (each $2 \mathrm{H}, \mathrm{t}, J=6.6 \mathrm{~Hz}, \mathrm{CH}_{2} \mathrm{CH}_{2}$ ), $7.41(1 \mathrm{H}$, dd, $J=7.4,8.4 \mathrm{~Hz}, \mathrm{Ar}-\mathrm{H}), 7.54(1 \mathrm{H}, \mathrm{dd}, J=6.8,7.4$ $\mathrm{Hz}, \mathrm{Ar}-\mathrm{H}), 7.60(1 \mathrm{H}, \mathrm{d}, J=8.4 \mathrm{~Hz}, \mathrm{Ar}-\mathrm{H}), 8.00(1 \mathrm{H}, \mathrm{d}, J=6.8 \mathrm{~Hz}, \mathrm{Ar}-\mathrm{H}) .{ }^{13} \mathrm{C}$ NMR $(100 \mathrm{MHz}$, $\left.\mathrm{CDCl}_{3}\right), \delta_{\mathrm{C}} 28.9\left(\mathrm{CH}_{2} \mathrm{Br}\right), 49.3\left(\mathrm{CH}_{2} \mathrm{~N}\right), 109.2,120.2,124.2,127.8(4 \mathrm{x} \mathrm{Ar}-\mathrm{CH}), 133.3,145.8(2$ x Ar-C). MS, $m / z=227 / 225\left(\mathrm{M}^{+}\right), 146,132,119$. HRMS: Calcd for $\mathrm{C}_{8} \mathrm{H}_{8}{ }^{79} \mathrm{BrN}_{3}: \mathrm{M}^{+}$224.9902; found: $\mathrm{M}^{+}$224.9902. Anal. Calcd for $\mathrm{C}_{8} \mathrm{H}_{8} \mathrm{BrN}_{3}$ : C, 42.50; H, 3.57; N, 18.59\%, Found: C, 42.23; H, 3.78; N, 18.22\%. $N^{2}$-(2-Bromoethyl)benzotriazole: yellow crystals (2.13 g, 56\%), m.p. 62-64 ${ }^{\circ} \mathrm{C}$ (lit., ${ }^{15}$ m.p. 59-60 $\left.{ }^{\circ} \mathrm{C}\right)$, IR $\left(v_{\max }, \mathrm{NaCl} / \mathrm{cm}^{-1}\right)$ 2960, 1648, 1583, 1454, 1284. ${ }^{1} \mathrm{H}$ NMR (400 $\mathrm{MHz}, \mathrm{CDCl}_{3}$ ), $\delta_{\mathrm{H}} 3.98,5.12$ (each $2 \mathrm{H}, \mathrm{t}, J=6.7 \mathrm{~Hz}, \mathrm{CH}_{2} \mathrm{CH}_{2}$ ), 7.42, 7.88 (each $2 \mathrm{H}, \mathrm{m}, \mathrm{Ar}-\mathrm{H}$ ). ${ }^{13} \mathrm{C}$ NMR $\left(100 \mathrm{MHz}, \mathrm{CDCl}_{3}\right), \delta_{\mathrm{C}} 28.1\left(\mathrm{CH}_{2} \mathrm{Br}\right), 57.3\left(\mathrm{CH}_{2} \mathrm{~N}\right), 118.2,126.7(\mathrm{Ar}-\mathrm{CH}), 144.5(\mathrm{Ar}-$ C). MS, $m / z=227 / 225\left(\mathrm{M}^{+}\right), 146,132,119$. HRMS: Calcd for $\mathrm{C}_{8} \mathrm{H}_{8}{ }^{79} \mathrm{BrN}_{3}: \mathrm{M}^{+} 224.9902$; found: $\mathrm{M}^{+}$224.9902. Anal. Calcd for $\mathrm{C}_{8} \mathrm{H}_{8} \mathrm{BrN}_{3}$ : C, 42.50; H, 3.57; N, 18.59\%, Found: C, 42.97; H, 3.24; N, 18.62\%. 
$\boldsymbol{N}^{\mathbf{1}}$-(2-Chloroethyl)benzotriazole. Prepared according to method $\mathrm{B}$, using benzotriazole (2.00 g, $16.81 \mathrm{mmol}), 1,2$-dichloroethane $(70 \mathrm{~mL})$, tetrabutylammonium iodide $(0.10 \mathrm{~g}, 5 \% \mathrm{w} / \mathrm{w}), \mathrm{KOH}$ (5.65 g, $0.10 \mathrm{~mol})$ and $\mathrm{K}_{2} \mathrm{CO}_{3}(5.80 \mathrm{~g}, 0.04 \mathrm{~mol})$. Work up gave the title compound as white crystals (2.01 g, 66\%), m.p. $118{ }^{\circ} \mathrm{C}$ (lit., ${ }^{16}$ m.p. 108-109 ${ }^{\circ} \mathrm{C}$ ), IR ( $v_{\max }, \mathrm{NaCl} / \mathrm{cm}^{-1}$ ) 2938, 1638, 1457, 1316, 1274, 1228, 1164. ${ }^{1} \mathrm{H}$ NMR (400 MHz, $\mathrm{CDCl}_{3}$ ), $\delta_{\mathrm{H}} 4.06,4.96$ (each 2H, t, $J=6.2$ $\left.\mathrm{Hz}, \mathrm{CH}_{2} \mathrm{CH}_{2}\right), 7.41(1 \mathrm{H}, \mathrm{t}, J=8.4 \mathrm{~Hz}, \mathrm{Ar}-\mathrm{H}), 7.55$ (1H, dd, $\left.J=6.8,8.4 \mathrm{~Hz}, \mathrm{Ar}-\mathrm{H}\right), 7.61$ (1H, d, $J$ $=6.8 \mathrm{~Hz}, \mathrm{Ar}-\mathrm{H}), 8.10(1 \mathrm{H}, \mathrm{d}, J=8.4 \mathrm{~Hz}, \mathrm{Ar}-\mathrm{H}) .{ }^{13} \mathrm{C} \mathrm{NMR}\left(100 \mathrm{MHz}, \mathrm{CDCl}_{3}\right), \delta_{\mathrm{C}} 42.3\left(\mathrm{CH}_{2} \mathrm{Cl}\right)$, $49.5\left(\mathrm{CH}_{2} \mathrm{~N}\right)$, 109.3, 120.2, 124.1, 127.8 (4 x Ar-CH), 133.5, 145.9 (2 x Ar-C). MS, m/z = 182 $\left(\mathrm{MH}^{+}\right)$, 146, 131, 119. HRMS: Calcd for $\mathrm{C}_{8} \mathrm{H}_{8}{ }^{35} \mathrm{ClN}_{3}: \mathrm{MH}^{+}$182.0479; found: $\mathrm{MH}^{+} 182.0478$. None of the isomeric $N^{2}$-(2-chloroethyl)benzotriazole was isolated.

$N^{1}$-(2-Iodoethyl)benzotriazole (9e). Prepared according to general procedure C, using $N^{1}$-(2bromoethyl)benzotriazole (1.00 g, $4.42 \mathrm{mmol})$ and dry NaI (3.32 g, $22.13 \mathrm{mmol})$ in dry acetone $(100 \mathrm{~mL})$. Work up gave the title compound as a pale yellow solid $(1.15 \mathrm{~g}, 95 \%), \mathrm{m} . \mathrm{p} .106{ }^{\circ} \mathrm{C}$, IR $\left(v_{\max }, \mathrm{NaCl} / \mathrm{cm}^{-1}\right)$ 3018, 2350, 1722, 1613, 1435, 1311. ${ }^{1} \mathrm{H}$ NMR (400 MHz, $\left.\mathrm{CDCl}_{3}\right), \delta_{\mathrm{H}} 3.67$, 5.02 (each 2H, t, $\left.J=7.4 \mathrm{~Hz}, \mathrm{CH}_{2} \mathrm{CH}_{2}\right), 7.36(1 \mathrm{H}, \mathrm{d}, J=6.6 \mathrm{~Hz}, \mathrm{Ar}-\mathrm{H}), 7.48(2 \mathrm{H}, \mathrm{m}, \mathrm{Ar}-\mathrm{H}), 8.04$ $(1 \mathrm{H}, \mathrm{d}, J=8.4 \mathrm{~Hz}, \mathrm{Ar}-\mathrm{H}) .{ }^{13} \mathrm{C}$ NMR $\left(100 \mathrm{MHz}, \mathrm{CDCl}_{3}\right), \delta_{\mathrm{C}} 30.2\left(\mathrm{CH}_{2} \mathrm{I}\right), 50.8\left(\mathrm{CH}_{2} \mathrm{~N}\right), 109.5$, 120.8, 124.9, 128.3 (4 x Ar-CH), 133.8, 146.4 (2 x Ar-C). MS, $m / z=274\left(\mathrm{MH}^{+}\right), 146,132,118$. HRMS: Calcd for $\mathrm{C}_{8} \mathrm{H}_{8} \mathrm{IN}_{3}$ : $\mathrm{MH}^{+}$273.9838; found: $\mathrm{MH}^{+} 273.9836$.

Also prepared according to general procedure C, but using $N^{1}$-(2-chloroethyl)benzotriazole (2.00 g, $11.02 \mathrm{mmol})$ and dry $\mathrm{NaI}(8.22 \mathrm{~g}, 54.80 \mathrm{mmol})$ in dry acetone $(100 \mathrm{~mL})$. Work up gave the title compound as a pale yellow solid (2.82 g, 94\%). Data as for material prepared using the bromoethyl derivative.

$\boldsymbol{N}^{\mathbf{2}}$-(2-Iodoethyl)benzotriazole (9f). Prepared according to general procedure C, using $N^{2}$-(2bromoethyl)benzotriazole (2.10 g, $9.29 \mathrm{mmol})$ and dry $\mathrm{NaI}(6.96 \mathrm{~g}, 46.40 \mathrm{mmol})$ in dry acetone $(100 \mathrm{~mL})$. Work up gave the title compound as a pale yellow solid (2.46 g, 97\%), m.p. 66-69 ${ }^{\circ} \mathrm{C}$, IR $\left(v_{\max }, \mathrm{NaCl} / \mathrm{cm}^{-1}\right.$ ) 3030, 1636, 1439, 1234. ${ }^{1} \mathrm{H}$ NMR (400 MHz, $\mathrm{CDCl}_{3}$ ), $\delta_{\mathrm{H}} 3.75,5.09$ (each $\left.2 \mathrm{H}, \mathrm{t}, J=7.3 \mathrm{~Hz}, \mathrm{CH}_{2} \mathrm{CH}_{2}\right), 7.42(2 \mathrm{H}, \mathrm{m}, \mathrm{Ar}-\mathrm{H}), 7.88(2 \mathrm{H}, \mathrm{m}, \mathrm{Ar}-\mathrm{H}) .{ }^{13} \mathrm{C} \mathrm{NMR}(100 \mathrm{MHz}$, $\left.\mathrm{CDCl}_{3}\right), \delta_{\mathrm{C}} 31.3\left(\mathrm{CH}_{2} \mathrm{I}\right), 59.3\left(\mathrm{CH}_{2} \mathrm{~N}\right), 119.2,127.8(2 \times \mathrm{Ar}-\mathrm{CH}), 145.5$ (Ar-C). MS, $\mathrm{m} / \mathrm{z}=273$ $\left(\mathrm{M}^{+}\right), 146,131,120$. HRMS: Calcd for $\mathrm{C}_{8} \mathrm{H}_{8} \mathrm{IN}_{3}: \mathrm{M}^{+}$272.9765; found: $\mathrm{M}^{+} 272.9757$.

\section{$N^{6}$-(tert-Butoxycarbonyl)adenine (6-tert-butoxycarbonylaminopurine)}

To a dry round-bottomed flask were added adenine (2.00 g, $14.81 \mathrm{mmol})$, di-tert-butyl dicarbonate (3.23 g, $14.82 \mathrm{mmol}$ ) and diisopropylethylamine (3.84 mL, $2.85 \mathrm{~g}, 22.09 \mathrm{mmol}$ ) in dry $\mathrm{CH}_{2} \mathrm{Cl}_{2}(100 \mathrm{~mL})$, and the mixture stirred for $2 \mathrm{~h}$ at $20{ }^{\circ} \mathrm{C}$. The solution was washed with water $(3 \times 20 \mathrm{~mL})$, dried $\left(\mathrm{MgSO}_{4}\right)$, filtered and the solvent removed under reduced pressure to give the title compound (3.46 g, quantitative yield), m.p. 280-282 ${ }^{\circ} \mathrm{C}$ (lit., ${ }^{17}$ m.p. 280-300 ${ }^{\circ} \mathrm{C}$ ), IR $\left(v_{\max }, \mathrm{NaCl} / \mathrm{cm}^{-1}\right)$ 3174, 2922, 1697, 1437, 1386. ${ }^{1} \mathrm{H}$ NMR (400 MHz, $\left.\mathrm{CDCl}_{3}\right), \delta_{\mathrm{H}} 1.71(9 \mathrm{H}, \mathrm{s}$, $\left.\mathrm{C}\left(\mathrm{CH}_{3}\right)_{3}\right), 1.85$ (1H, br, 9-NH), 5.78 (1H, br, NHBoc), 8.51, 8.52 (each 1H, s, Ar-H). ${ }^{13} \mathrm{C}$ NMR (100 MHz, $\left.\mathrm{CDCl}_{3}\right), \delta_{\mathrm{C}} 27.9\left(\mathrm{C}\left(\mathrm{CH}_{3}\right)_{3}\right), 37.6\left(\mathrm{C}\left(\mathrm{CH}_{3}\right)_{3}\right), 144.6(\mathrm{Ar}-\mathrm{CH}), 147.9,151.8$ (2 x Ar-C), 
155.4 (Ar-CH), 161.5 (Ar-C), 204.6 (C=O). MS, m/z = $235\left(\mathrm{M}^{+}\right), 134$, 119, 90. HRMS: Calcd for $\mathrm{C}_{10} \mathrm{H}_{13} \mathrm{~N}_{5} \mathrm{O}_{2}$ : $\mathrm{M}^{+}$235.1069; found: $\mathrm{M}^{+} 235.1069$.

\section{$N^{9}$-(2-Bromoethyl)-6-tert-butoxycarbonylaminopurine}

Prepared according to method A, using 6-tert-butoxycarbonylaminopurine (3.45 g, $14.68 \mathrm{mmol}$ ), triphenylphosphine (4.62 g, $17.63 \mathrm{mmol})$ and 2-bromoethanol (1.24 mL, $2.18 \mathrm{~g}, 17.48 \mathrm{mmol})$ in dry dioxane $(100 \mathrm{~mL})$ with DIAD $(3.47 \mathrm{~mL}, 3.56 \mathrm{~g}, 17.64 \mathrm{mmol})$. After workup this yielded the title compound as colourless crystals (1.88 g, 37\%), m.p. $197{ }^{\circ} \mathrm{C} .{ }^{1} \mathrm{H}$ NMR (400 $\mathrm{MHz}, \mathrm{CDCl}_{3}$ ), $\delta_{\mathrm{H}} 1.63\left(9 \mathrm{H}, \mathrm{s}, \mathrm{C}\left(\mathrm{CH}_{3}\right)_{3}\right)$, 3.82, 4.41 (each $\left.2 \mathrm{H}, \mathrm{t}, J=7.8 \mathrm{~Hz}, \mathrm{CH}_{2} \mathrm{CH}_{2}\right), 6.30(1 \mathrm{H}, \mathrm{br}, \mathrm{NH}), 8.19$, 8.47 (each $1 \mathrm{H}, \mathrm{s}, \mathrm{Ar}-\mathrm{H}) .{ }^{13} \mathrm{C} \mathrm{NMR}\left(100 \mathrm{MHz}, \mathrm{CDCl}_{3}\right), \delta_{\mathrm{C}} 31.9\left(\mathrm{C}\left(\mathrm{CH}_{3}\right)_{3}\right), 38.2\left(\mathrm{C}_{\left.\left(\mathrm{CH}_{3}\right)_{3}\right),} 38.6\right.$ $\left(\mathrm{CH}_{2} \mathrm{Br}\right), 51.9\left(\mathrm{CH}_{2} \mathrm{~N}\right), 137.5(\mathrm{Ar}-\mathrm{CH}), 147.2,149.3$ (2 x Ar-C), 157.8 (Ar-CH), 161.2 (Ar-C) $216.4(\mathrm{C}=\mathrm{O})$. MS, $m / z=343 / 341\left(\mathrm{M}^{+}\right)$, 241, 133, 118, 90. LC-MS: Calcd for $\mathrm{C}_{12} \mathrm{H}_{16}{ }^{79} \mathrm{BrN}_{5} \mathrm{O}_{2}$ : $\mathrm{M}^{+}$341.05; found: $\mathrm{M}^{+} 341.05$.

$N^{9}$-(2-Iodoethyl)-6-tert-butoxycarbonylaminopurine $(\mathbf{9 g})$. Prepared according to general procedure C, using $N^{9}$-(2-bromoethyl)-6-tert-butoxycarbonylaminopurine (1.60 g, $4.68 \mathrm{mmol}$ ) and dry $\mathrm{NaI}$ (3.50 g, $23.33 \mathrm{mmol})$ in dry acetone $(100 \mathrm{~mL})$. Work up gave the title compound as a pale yellow oil $(0.92 \mathrm{~g}, 51 \%)$, IR $\left(v_{\max }, \mathrm{NaCl} / \mathrm{cm}^{-1}\right) 3419,1642,1516,1436,1373,1277 .{ }^{1} \mathrm{H}$ NMR (400 MHz, $\left.\mathrm{CDCl}_{3}\right), \delta_{\mathrm{H}} 1.39\left(9 \mathrm{H}, \mathrm{s}, \mathrm{C}\left(\mathrm{CH}_{3}\right)_{3}\right), 3.57,4.51$ (each $2 \mathrm{H}, \mathrm{t}, J=6.6 \mathrm{~Hz}, \mathrm{CH}_{2} \mathrm{CH}_{2}$ ), $5.99(1 \mathrm{H}$, br, NH), 7.80, 8.34 (each $1 \mathrm{H}, \mathrm{s}, \mathrm{Ar}-\mathrm{CH}) .{ }^{13} \mathrm{C} \mathrm{NMR}\left(100 \mathrm{MHz}, \mathrm{CDCl}_{3}\right), \delta_{\mathrm{C}} 28.2$ $\left(\mathrm{C}\left(\mathrm{CH}_{3}\right)_{3}\right), 29.9\left(\mathrm{CH}_{2} \mathrm{I}\right), 38.2\left(\mathrm{C}\left(\mathrm{CH}_{3}\right)_{3}\right), 51.7\left(\mathrm{CH}_{2} \mathrm{~N}\right), 139.1(\mathrm{Ar}-\mathrm{CH}), 148.7,150.7$ (2 x Ar-C), 155.8 (Ar-CH), 163.9 (Ar-C), $213.4(\mathrm{C}=\mathrm{O}) . \mathrm{MS}, \mathrm{m} / \mathrm{z}=389\left(\mathrm{M}^{+}\right), 288,134,118$, 90. HRMS: Calcd for $\mathrm{C}_{12} \mathrm{H}_{16} \mathrm{IN}_{5} \mathrm{O}_{2}$ : $\mathrm{M}^{+}$389.0351; found: $\mathrm{M}^{+}$389.0375.

$N^{9}$-(2-Bromoethyl)purine. Prepared according to method A, using purine (0.50 g, $\left.4.16 \mathrm{mmol}\right)$, triphenylphosphine $(1.32 \mathrm{~g}, 5.04 \mathrm{mmol})$ and 2-bromoethanol $(0.36 \mathrm{~mL}, 0.63 \mathrm{~g}, 5.07 \mathrm{mmol})$ in dry dioxane $(100 \mathrm{~mL})$ and DIAD $(0.99 \mathrm{~mL}, 1.02 \mathrm{~g}, 5.03 \mathrm{mmol})$. After workup this yielded the title compound as a brown solid (0.62 g, 73\%), m.p. 188-190 ${ }^{\circ} \mathrm{C}$ (lit., ${ }^{18}$ m.p. $195{ }^{\circ} \mathrm{C}$ ), IR ( $v_{\max }$, $\mathrm{NaCl} / \mathrm{cm}^{-1}$ ) 3289, 2979, 1700, 1496, 1456, 1267, 1219. ${ }^{1} \mathrm{H}$ NMR (400 MHz, $\mathrm{CDCl}_{3}$ ), $\delta_{\mathrm{H}} 3.68$, 4.58 (each $2 \mathrm{H}, \mathrm{t}, \mathrm{J}=5.5 \mathrm{~Hz}, \mathrm{CH}_{2} \mathrm{CH}_{2}$ ), 8.14, 8.74, 8.92 (each $1 \mathrm{H}, \mathrm{s}, \mathrm{Ar}-\mathrm{H}$ ). ${ }^{13} \mathrm{C} \mathrm{NMR}(100 \mathrm{MHz}$, $\left.\mathrm{CDCl}_{3}\right), \delta_{\mathrm{C}} 55.0\left(\mathrm{CH}_{2} \mathrm{Br}\right), 60.3\left(\mathrm{CH}_{2} \mathrm{~N}\right), 138.8,141.6(2$ x Ar-CH), 143.7 (Ar-C), $155.6(\mathrm{Ar}-\mathrm{CH})$, 160.8 (Ar-C). MS, $m / z=228 / 226\left(M^{+}\right), 144,117,91$. HRMS: Calcd for $\mathrm{C}_{7} \mathrm{H}_{7}{ }^{81} \mathrm{BrN}_{4}$ requires: $\mathrm{M}^{+}$ 227.9834; found: $\mathrm{M}^{+} 227.9851$.

$N^{9}$-(2-Chloroethyl)purine. Prepared according to method B, using purine (0.50 g, $\left.4.16 \mathrm{mmol}\right)$ in 1,2-dichloroethane $(50 \mathrm{~mL})$ with tetrabutylammonium iodide $(0.03 \mathrm{~g}, 5 \% \mathrm{w} / \mathrm{w}), \mathrm{KOH}$ (1.41 g, $25.18 \mathrm{mmol})$ and $\mathrm{K}_{2} \mathrm{CO}_{3}(1.45 \mathrm{~g}, 10.51 \mathrm{mmol})$. Purification gave the title compound as a yellow oil (0.39 g, 59\%), IR ( $\left.v_{\max }, \mathrm{NaCl} / \mathrm{cm}^{-1}\right)$ 3082, 2962, 1594, 1502, 1408, 1305, 1200. ${ }^{1} \mathrm{H}$ NMR (400 MHz, $\mathrm{CDCl}_{3}$ ), $\delta_{\mathrm{H}}$ 3.91, 4.59 (each 2H, t, $J=5.6 \mathrm{~Hz}, \mathrm{CH}_{2} \mathrm{CH}_{2}$ ), 8.16, 8.91, 9.09 (each 1H, s, $\mathrm{Ar}-\mathrm{H}) .{ }^{13} \mathrm{C}$ NMR $\left(100 \mathrm{MHz}, \mathrm{CDCl}_{3}\right), \delta_{\mathrm{C}} 42.2\left(\mathrm{CH}_{2} \mathrm{Cl}\right), 45.6\left(\mathrm{CH}_{2} \mathrm{~N}\right), 134.1(\mathrm{Ar}-\mathrm{C}), 145.8,148.7$ (2 x Ar-CH), 151.1 (Ar-C), 153.2 (Ar-CH). MS, m/z = $183\left(\mathrm{MH}^{+}\right), 144,117,91$. HRMS: Calcd for $\mathrm{C}_{7} \mathrm{H}_{7}{ }^{35} \mathrm{ClN}_{4}$ : $\mathrm{MH}^{+}$183.0432; found: $\mathrm{MH}^{+} 183.0458$. 
$N^{9}$-(2-Iodoethyl)purine (9h). Prepared according to general procedure C, using $N^{9}$-(2bromoethyl)purine $(0.25 \mathrm{~g}, 1.10 \mathrm{mmol})$ and dry $\mathrm{NaI}(0.92 \mathrm{~g}, 6.13 \mathrm{mmol})$ in dry acetone (100 $\mathrm{mL})$. Work up gave the title compound as a pale yellow oil $(0.24 \mathrm{~g}, 79 \%)$, IR $\left(v_{\max }\left(\mathrm{NaCl} / \mathrm{cm}^{-1}\right)\right.$ 2983, 1642, 1496, 1436, 1312. ${ }^{1} \mathrm{H}$ NMR (400 MHz, $\mathrm{CDCl}_{3}$ ), $\delta_{\mathrm{H}} 3.61,4.66$ (each $2 \mathrm{H}, \mathrm{t}, J=6.5$ $\mathrm{Hz}, \mathrm{CH}_{2} \mathrm{CH}_{2}$ ), 8.16, 8.95, 9.14 (each $\left.1 \mathrm{H}, \mathrm{s}, \mathrm{Ar}-\mathrm{H}\right) .{ }^{13} \mathrm{C}$ NMR (100 MHz, $\left.\mathrm{CDCl}_{3}\right), \delta_{\mathrm{C}} 23.2\left(\mathrm{CH}_{2} \mathrm{I}\right)$, $45.9\left(\mathrm{CH}_{2} \mathrm{~N}\right), 135.0(\mathrm{Ar}-\mathrm{C}), 144.9,148.8$, (2 x Ar-CH), 152.0 (Ar-C), $152.6(\mathrm{Ar}-\mathrm{CH}) . \mathrm{MS}, \mathrm{m} / \mathrm{z}=$ $275\left(\mathrm{MH}^{+}\right), 145,117,90$. HRMS: Calcd for $\mathrm{C}_{7} \mathrm{H}_{7} \mathrm{IN}_{4}: \mathrm{MH}^{+}$274.9790; found $\mathrm{MH}^{+} 274.9768$.

$\mathrm{N}$-(3-Chloropropyl)indole. Prepared according to method B, using indole (2.00 g, $17.09 \mathrm{mmol}$ ), 1,3-dichloropropane $\left(150 \mathrm{~cm}^{3}\right)$, tetrabutylammonium iodide $(0.10 \mathrm{~g}, 5 \% \mathrm{w} / \mathrm{w}), \mathrm{KOH}(5.75 \mathrm{~g}$, $0.10 \mathrm{~mol})$ and $\mathrm{K}_{2} \mathrm{CO}_{3}(5.90 \mathrm{~g}, 0.04 \mathrm{~mol})$. Purification by flash column chromatography gave title compound as a brown oil (2.01 g, 61\%), IR $\left(v_{\max }, \mathrm{NaCl} / \mathrm{cm}^{-1}\right) 3054,2970,2862,2218,1718$, 1589, 1437, 1191. ${ }^{1} \mathrm{H}$ NMR (400 MHz, CDCl $), \delta_{\mathrm{H}} 2.35\left(2 \mathrm{H}, \mathrm{q}, J=6.4 \mathrm{~Hz}, \mathrm{CH}_{2}\right), 3.55(2 \mathrm{H}, \mathrm{t}, J=$ $6.4 \mathrm{~Hz}, \mathrm{CH}_{2} \mathrm{Cl}$ ), $4.42\left(2 \mathrm{H}, \mathrm{t}, J 6.4=\mathrm{Hz}, \mathrm{CH}_{2} \mathrm{~N}\right.$ ), 6.75, 7.28 (each $1 \mathrm{H}, \mathrm{d}, J=3.1 \mathrm{~Hz}$, indole-CH), 7.38, 7.46 (each 1H, dd, $J=7.1,8.1 \mathrm{~Hz}, \mathrm{Ar}-\mathrm{H}$ ), 7.56, 7.80 (each $1 \mathrm{H}, \mathrm{d}, J=8.1 \mathrm{~Hz}, \mathrm{Ar}-\mathrm{H}$ ). ${ }^{13} \mathrm{C}$ NMR $\left(100 \mathrm{MHz}, \mathrm{CDCl}_{3}\right), \delta_{\mathrm{C}} 30.4\left(\mathrm{CH}_{2}\right), 42.2\left(\mathrm{CH}_{2} \mathrm{Cl}\right), 43.1\left(\mathrm{CH}_{2} \mathrm{~N}\right), 101.7,109.5(2 \mathrm{x}$ indole$\mathrm{CH}), 117.4,119.7,121.6,122.1$ (4 x Ar-CH), 133.7, 136.1 (2 x Ar-C). MS, m/z = $194\left(\mathrm{MH}^{+}\right)$, 158, 118, 91. HRMS: Calcd for $\mathrm{C}_{11} \mathrm{H}_{12}{ }^{35} \mathrm{ClN}$ : $\mathrm{MH}^{+}$194.0731; found: $\mathrm{MH}^{+}$194.0723.

$N^{1}$-(3-Bromopropyl)benzimidazole. Prepared according to method A, using benzimidazole (1.00 g, $8.47 \mathrm{mmol})$, triphenylphosphine (2.66 g, $10.15 \mathrm{mmol})$ and 3-bromopropan-1-ol (0.92 $\mathrm{mL}, 1.41 \mathrm{~g}, 10.15 \mathrm{mmol})$ in dry dioxane $(100 \mathrm{~mL})$ at $5{ }^{\circ} \mathrm{C}$ with DIAD $(4.00 \mathrm{~mL}, 4.11 \mathrm{~g}, 20.33$ mmol) to yield the title compound as an oil $(1.58 \mathrm{~g}, 78 \%) .{ }^{1} \mathrm{H}$ NMR $\left(400 \mathrm{MHz}, \mathrm{CDCl}_{3}\right), \delta_{\mathrm{H}} 2.10$ $\left(2 \mathrm{H}, \mathrm{m}, \mathrm{CH}_{2}\right), 3.54\left(2 \mathrm{H}, \mathrm{t}, J=6.3 \mathrm{~Hz}, \mathrm{CH}_{2} \mathrm{Br}\right), 4.20\left(2 \mathrm{H}, \mathrm{t}, J=6.6 \mathrm{~Hz}, \mathrm{CH}_{2} \mathrm{~N}\right), 7.23,7.74$ (each $2 \mathrm{H}, \mathrm{m}, \mathrm{Ar}-\mathrm{H},), 7.83(1 \mathrm{H}, \mathrm{s}, 2-\mathrm{CH}) .{ }^{13} \mathrm{C}$ NMR $\left(100 \mathrm{MHz}, \mathrm{CDCl}_{3}\right), \delta_{\mathrm{C}} 27.0\left(\mathrm{CH}_{2}\right), 29.6\left(\mathrm{CH}_{2} \mathrm{Br}\right)$, $34.0\left(\mathrm{CH}_{2} \mathrm{~N}\right), 109.6$ (2-CH), 120.5, 122.1, 123.0, (3 x Ar-CH), 133.7 (Ar-C), 142.9 (Ar-CH), 143.9 (Ar-C). MS, $m / z=241 / 239\left(\mathrm{MH}^{+}\right), 160,118,104$, 90. HRMS: Calcd for $\mathrm{C}_{10} \mathrm{H}_{11}{ }^{79} \mathrm{BrN}_{2}$ : $\mathrm{MH}^{+}$239.0179; found: $\mathrm{MH}^{+} 239.0150$.

$N^{1}$-(3-Chloropropyl)benzimidazole. Prepared according to method B, using benzimidazole $(1.00 \mathrm{~g}, 8.47 \mathrm{mmol})$ in 1,3-dichloropropane $(70 \mathrm{~mL})$ with tetrabutylammonium iodide $(0.05 \mathrm{~g}$, $5 \% \mathrm{w} / \mathrm{w}$ ), $\mathrm{KOH}$ (2.85 g, $50.89 \mathrm{mmol}$ ) and $\mathrm{K}_{2} \mathrm{CO}_{3}$ (2.93 g, $\left.21.23 \mathrm{mmol}\right)$. After stirring for $8 \mathrm{~h}$, work up gave title compound as an oil $(1.04 \mathrm{~g}, 62 \%) .{ }^{1} \mathrm{H}$ NMR $\left(400 \mathrm{MHz}, \mathrm{CDCl}_{3}\right), \delta_{\mathrm{H}} 2.23(2 \mathrm{H}$, m, $\mathrm{CH}_{2}$ ), $3.38\left(2 \mathrm{H}, \mathrm{t}, J=6.5 \mathrm{~Hz}, \mathrm{CH}_{2} \mathrm{Cl}\right), 4.32\left(2 \mathrm{H}, \mathrm{t}, J=6.5 \mathrm{~Hz}, \mathrm{CH}_{2} \mathrm{~N}\right), 7.23,7.74$ (each $2 \mathrm{H}$, m, Ar-H,), $7.83(1 \mathrm{H}, \mathrm{s}, 2-\mathrm{CH}) .{ }^{13} \mathrm{C}$ NMR $\left(100 \mathrm{MHz}, \mathrm{CDCl}_{3}\right), \delta_{\mathrm{C}} 22.7\left(\mathrm{CH}_{2}\right), 32.2\left(\mathrm{CH}_{2} \mathrm{Cl}\right), 46.4$ $\left(\mathrm{CH}_{2} \mathrm{~N}\right), 102.8$ (2-CH), 121.1, 128.9, 129.2, 133.6 (4 x Ar-CH), 138.2, 147.3 (2 x ArC). MS, m/z $=194\left(\mathrm{M}^{+}\right)$, 159, 118, 104, 90. HRMS: Calcd for $\mathrm{C}_{10} \mathrm{H}_{11}{ }^{35} \mathrm{ClN}_{2}$ : $\mathrm{M}^{+}$194.0611; found: $\mathrm{M}^{+}$ 194.0609.

\section{Attempted preparation of $N^{1}$-(3-iodopropyl)benzimidazole (10c): Quaternary salt (12a)}

Attempted according to general procedure C, using $N^{1}$-(3-bromopropyl)benzimidazole and (1.00 g, $4.18 \mathrm{mmol})$ and dry NaI (3.15 g, $21.00 \mathrm{mmol})$ in dry acetone $(100 \mathrm{~mL})$, and also by using $N^{1}$ (3-chloropropyl)benzimidazole (1.00 g, $5.14 \mathrm{mmol})$ and dry NaI (3.86 g, $25.73 \mathrm{mmol})$ in dry 
acetone $(100 \mathrm{~mL})$. Work up in both cases did not give the title compound, but gave what is provisionally assigned as the dimeric quaternary salt 12a. ${ }^{1} \mathrm{H} \mathrm{NMR}\left(250 \mathrm{MHz}, \mathrm{CDCl}_{3}\right), \delta_{\mathrm{H}} 1.90$ (2H, m, $\mathrm{CH}_{2}$ ), 4.90 (4H, t, $\left.J=6.9 \mathrm{~Hz}, 2 \mathrm{x} \mathrm{CH}_{2}\right), 7.22-7.78$ (4H, m, Ar-H), 7.95 (1H, s, 2-CH). LC-MS: Calcd for double quaternary cation $\mathrm{C}_{20} \mathrm{H}_{22} \mathrm{~N}_{4}$ : $\mathrm{M}^{2+} 159.09$, [M-H] ${ }^{+}$317.18; found: $\mathrm{M}^{2+}$ 158.93, [M-H] $]^{+} 317.30$.

$\boldsymbol{N}^{\mathbf{1}}$-(3-Bromopropyl)benzotriazole. Prepared according to method A, using benzotriazole (4.00 g, $33.61 \mathrm{mmol})$, triphenylphosphine (10.57 g, $40.34 \mathrm{mmol}$ ) and 3-bromopropan-1-ol (3.64 mL, $5.61 \mathrm{~g}, 40.33 \mathrm{mmol})$ in dry dioxane $(100 \mathrm{~mL})$ with DIAD (7.93 mL, $8.14 \mathrm{~g}, 40.32 \mathrm{mmol})$. After workup this yielded the title compound as a yellow oil (3.15 g, 78\%), IR ( $\left.v_{\max }, \mathrm{NaCl} / \mathrm{cm}^{-1}\right) 2900$, 1660, 1542, 1447, 1287. ${ }^{1} \mathrm{H}$ NMR (400 MHz, $\left.\mathrm{CDCl}_{3}\right), \delta_{\mathrm{H}} 2.33\left(2 \mathrm{H}, \mathrm{tt}, J=6.8,7.3 \mathrm{~Hz}, \mathrm{CH}_{2}\right) 3.28$ $\left(2 \mathrm{H}, \mathrm{t}, J=6.8 \mathrm{~Hz}, \mathrm{CH}_{2} \mathrm{Cl}\right), 4.75\left(2 \mathrm{H}, \mathrm{t}, J 7.3 \mathrm{~Hz}, \mathrm{CH}_{2} \mathrm{~N}\right), 7.37$ (1H, m, Ar-H), 7.46-7.58 (2H, m, Ar-H), $8.01(1 \mathrm{H}, \mathrm{d}, J=7.9 \mathrm{~Hz}, \mathrm{Ar}-\mathrm{H}) .{ }^{13} \mathrm{C} \mathrm{NMR}\left(100 \mathrm{MHz}, \mathrm{CDCl}_{3}\right), \delta_{\mathrm{C}} 27.8\left(\mathrm{CH}_{2}\right), 32.4$ $\left(\mathrm{CH}_{2} \mathrm{Cl}\right), 51.9\left(\mathrm{CH}_{2} \mathrm{~N}\right), 109.7,121.2,123.7,126.5$ (4 x Ar-CH), 136.3, 144.4 (2 x Ar-C). MS, m/z $=240\left(\mathrm{MH}^{+}\right)$, 160, 132, 119. HRMS: Calcd for $\mathrm{C}_{9} \mathrm{H}_{10}{ }^{79} \mathrm{BrN}_{3}: \mathrm{MH}^{+}$240.0131; found: $\mathrm{MH}^{+}$ 240.0153. None of the isomeric $N^{2}$-(3-bromopropyl)benzotriazole was isolated.

$N^{1}$ - and $\boldsymbol{N}^{2}$-(3-Chloropropyl)benzotriazole. Prepared according to method B, using benzotriazole (2.00 g, $16.81 \mathrm{mmol})$, 1,3-dichloropropane $\left(100 \mathrm{~cm}^{3}\right)$, tetrabutylammonium iodide (0.10 g, 5\% w/w), $\mathrm{KOH} \mathrm{(5.65} \mathrm{g,} 0.10 \mathrm{~mol})$ and $\mathrm{K}_{2} \mathrm{CO}_{3}(5.80 \mathrm{~g}, 0.04 \mathrm{~mol})$. Work up and chromatography gave the title compounds. $N^{1}$-(3-Chloropropyl)benzotriazole: cream oil (1.59 g, 52\%), IR ( $\left.v_{\max }, \mathrm{NaCl} / \mathrm{cm}^{-1}\right)$ 2879, 1687, 1523, 1438, 1202. ${ }^{1} \mathrm{H}$ NMR (400 MHz, $\left.\mathrm{CDCl}_{3}\right), \delta_{\mathrm{H}} 2.45$ $\left(2 \mathrm{H}, \mathrm{tt}, J=6.0,6.5 \mathrm{~Hz}, \mathrm{CH}_{2}\right) 3.49\left(2 \mathrm{H}, \mathrm{t}, J=6.0 \mathrm{~Hz}, \mathrm{CH}_{2} \mathrm{Cl}\right), 4.79\left(2 \mathrm{H}, \mathrm{t}, J=6.5 \mathrm{~Hz}, \mathrm{CH}_{2} \mathrm{~N}\right)$, 7.34, 7.48 (each 1H, t, $J=8.3 \mathrm{~Hz}$, Ar-H), 7.58, 8.03 (each $1 \mathrm{H}, \mathrm{d}, J=8.3 \mathrm{~Hz}, \mathrm{Ar}-\mathrm{H}$ ). ${ }^{13} \mathrm{C}$ NMR (100 MHz, $\left.\mathrm{CDCl}_{3}\right), \delta_{\mathrm{C}} 32.4\left(\mathrm{CH}_{2}\right), 41.5\left(\mathrm{CH}_{2} \mathrm{Cl}\right), 44.7\left(\mathrm{CH}_{2} \mathrm{~N}\right), 109.2,120.0,124.0,127.5(4 \mathrm{x}$ Ar-CH), 133.2, 145.9 (2 x ArC). MS, $m / z=196\left(\mathrm{MH}^{+}\right), 160,133,120$. HRMS: Calcd for $\mathrm{C}_{9} \mathrm{H}_{10}{ }^{35} \mathrm{ClN}_{3}$ : $\mathrm{MH}^{+}$196.0636; found: $\mathrm{MH}^{+}$196.0642. Anal. Calcd for $\mathrm{C}_{9} \mathrm{H}_{10} \mathrm{ClN}_{3}$ : C, 55.25; $\mathrm{H}$, 5.15; N, 21.48\%; Found: C, 55.86; H, 4.74; N, 21.82\%. $N^{2}$-(3-Chloropropyl)benzotriazole: yellow oil (0.72 g, 24\%), IR ( $\left.v_{\max }, \mathrm{NaCl} / \mathrm{cm}^{-1}\right)$ 2901, 1732, 1647, 1519, 1468, 1232. ${ }^{1} \mathrm{H}$ NMR (400 MHz, $\left.\mathrm{CDCl}_{3}\right), \delta_{\mathrm{H}} 2.53\left(2 \mathrm{H}, \mathrm{tt}, J=6.2,6.5 \mathrm{~Hz}, \mathrm{CH}_{2}\right), 3.56\left(2 \mathrm{H}, \mathrm{t}, J=6.2 \mathrm{~Hz}, \mathrm{CH}_{2} \mathrm{Cl}\right), 4.89$ $\left(2 \mathrm{H}, \mathrm{t}, J=6.5 \mathrm{~Hz}, \mathrm{CH}_{2} \mathrm{~N}\right), 7.35,7.84$ (each $\left.2 \mathrm{H}, \mathrm{m}, \mathrm{Ar}-\mathrm{H}\right) .{ }^{13} \mathrm{C} \mathrm{NMR}\left(100 \mathrm{MHz}, \mathrm{CDCl}_{3}\right), \delta_{\mathrm{C}} 32.6$ $\left(\mathrm{CH}_{2}\right), 41.4\left(\mathrm{CH}_{2} \mathrm{Cl}\right), 53.4\left(\mathrm{CH}_{2} \mathrm{~N}\right), 117.9,126.4$ (2 x Ar-CH), 144.4 (Ar-C). MS, m/z = 195 $\left(\mathrm{M}^{+}\right)$, 146, 133, 119. HRMS: Calcd for $\mathrm{C}_{9} \mathrm{H}_{10}{ }^{35} \mathrm{ClN}_{3}$ : $\mathrm{M}^{+}$195.0563; found: $\mathrm{M}^{+}$195.0560. Anal. Calcd for $\mathrm{C}_{9} \mathrm{H}_{10} \mathrm{ClN}_{3}$ : C, 55.25; H, 5.15; N, 21.48\%; Found: C, 54.91; H, 5.24; N, 20.94\%.

$N^{1}$-(3-Iodopropyl)benzotriazole (10e). Prepared according to general procedure C, using $N^{1}$-(3chloropropyl)benzotriazole $(1.50 \mathrm{~g}, 7.67 \mathrm{mmol})$ and dry $\mathrm{NaI}(6.19 \mathrm{~g}, 41.27 \mathrm{mmol})$ in dry acetone $(100 \mathrm{~mL})$. Work up gave the title compound as a pale yellow solid (1.73 g, 77\%), m.p. 98-99 ${ }^{\circ} \mathrm{C}$, IR $\left(v_{\max }, \mathrm{NaCl} / \mathrm{cm}^{-1}\right)$ 2958, 1610, 1464, 1357, 1205. ${ }^{1} \mathrm{H}$ NMR (400 MHz, $\left.\mathrm{CDCl}_{3}\right), \delta_{\mathrm{H}} 2.44(2 \mathrm{H}$, tt, $\left.J=6.4,6.5 \mathrm{~Hz}, \mathrm{CH}_{2}\right), 3.12\left(2 \mathrm{H}, \mathrm{t}, J=6.5 \mathrm{~Hz}, \mathrm{CH}_{2} \mathrm{I}\right), 4.73\left(2 \mathrm{H}, \mathrm{t}, J=6.4 \mathrm{~Hz}, \mathrm{CH}_{2} \mathrm{~N}\right), 7.01,7.39$ (each $1 \mathrm{H}, \mathrm{t}, J=7.0 \mathrm{~Hz}, \mathrm{Ar}-\mathrm{H}), 7.44,7.68$ (each $1 \mathrm{H}, \mathrm{d}, J=8.1 \mathrm{~Hz}, \mathrm{Ar}-\mathrm{H}) .{ }^{13} \mathrm{C} \mathrm{NMR}(100 \mathrm{MHz}$, $\left.\mathrm{CDCl}_{3}\right), \delta_{\mathrm{C}} 3.5\left(\mathrm{CH}_{2} \mathrm{I}\right), 33.3\left(\mathrm{CH}_{2}\right), 45.9\left(\mathrm{CH}_{2} \mathrm{~N}\right), 109.3,118.3,121.5,124.5(4 \mathrm{x} \mathrm{Ar}-\mathrm{CH}), 127.0$, 
$136.8\left(2 \times\right.$ ArC). MS, $m / z=286\left([\mathrm{M}-\mathrm{H}]^{+}\right), 160,132,119$. HRMS: Calcd for $\mathrm{C}_{9} \mathrm{H}_{10} \mathrm{IN}_{3}:[\mathrm{M}-\mathrm{H}]^{+}$ 285.9849; found: [M-H] $]^{+} 285.9887$.

$N^{2}$-(3-Iodopropyl)benzotriazole (10f). Prepared according to general procedure C, using $N^{2}$-(3chloropropyl)benzimidazole (3.20 g, $16.37 \mathrm{mmol})$ and dry NaI (13.20 g, $88.00 \mathrm{mmol})$ in dry acetone $(100 \mathrm{~mL})$. Work up gave the title compound as a pale yellow oil $(2.94 \mathrm{~g}, 65 \%)$, IR ( $v_{\max }$, $\mathrm{NaCl} / \mathrm{cm}^{-1}$ ) 2962, 1615, 1448, 1278. ${ }^{1} \mathrm{H}$ NMR (400 MHz, $\left.\mathrm{CDCl}_{3}\right), \delta_{\mathrm{H}} 2.58(2 \mathrm{H}, \mathrm{tt}, J=6.4,6.7$ $\mathrm{Hz}, \mathrm{CH}_{2}$ ), $3.15\left(2 \mathrm{H}, \mathrm{t}, J=6.7 \mathrm{~Hz}, \mathrm{CH}_{2} \mathrm{I}\right), 4.81\left(2 \mathrm{H}, \mathrm{t}, J=6.4 \mathrm{~Hz}, \mathrm{CH}_{2} \mathrm{~N}\right), 7.37,7.82$ (each $2 \mathrm{H}, \mathrm{m}$, Ar-H). ${ }^{13} \mathrm{C}$ NMR (100 MHz, $\left.\mathrm{CDCl}_{3}\right), \delta_{\mathrm{C}} 4.2\left(\mathrm{CH}_{2} \mathrm{I}\right), 30.6\left(\mathrm{CH}_{2}\right), 50.9\left(\mathrm{CH}_{2} \mathrm{~N}\right), 115.7,128.2(2 \mathrm{x}$ Ar-CH), 138.1 (Ar-C). MS, $m / z=286, M^{+}, 160,133,119$. HRMS: Calcd for $\mathrm{C}_{9} \mathrm{H}_{10} \mathrm{IN}_{3}: \mathrm{M}^{+}$ 285.9849; found: $\mathrm{M}^{+} 285.9856$.

\section{Attempted preparation of $\boldsymbol{N}^{\mathbf{1}}$-(3-chlorobutyl)benzimidazole: quaternary salt (12b)}

Attempted according to method B, using benzimidazole (1.00 g, $8.47 \mathrm{mmol})$ in 1,4dichlorobutane $(70 \mathrm{~mL})$ with tetrabutylammonium iodide $(0.05 \mathrm{~g}, 5 \% \mathrm{w} / \mathrm{w}), \mathrm{KOH}$ (2.85 g, 50.89 mmol) and $\mathrm{K}_{2} \mathrm{CO}_{3}(2.30 \mathrm{~g}, 16.67 \mathrm{mmol})$. After stirring for $8 \mathrm{~h}$, work up did not give the title compound, but gave what is provisionally assigned as the dimeric quaternary salt $\mathbf{1 2 b} .{ }^{1} \mathrm{H}$ NMR $\left(400 \mathrm{MHz},\left(\mathrm{CD}_{3}\right)_{2} \mathrm{SO}\right), \delta_{\mathrm{H}} 2.08\left(4 \mathrm{H}, \mathrm{br}, 2 \times \mathrm{CH}_{2}\right), 4.57\left(4 \mathrm{H}, \mathrm{t}, J=5.6 \mathrm{~Hz}, 2 \times \mathrm{CH}_{2}\right), 7.67,8.34$ (each 2H, m, Ar-H), $9.77(1 \mathrm{H}, \mathrm{s}, 2-\mathrm{CH}) .{ }^{13} \mathrm{C} \mathrm{NMR}\left(100 \mathrm{MHz},\left(\mathrm{CD}_{3}\right)_{2} \mathrm{SO}\right), \delta_{\mathrm{C}} 24.9\left(\mathrm{CH}_{2}\right), 26.0$ $\left(\mathrm{CH}_{2}\right), 29.7\left(\mathrm{CH}_{2}\right), 46.2\left(\mathrm{CH}_{2} \mathrm{~N}\right), 113.8(2-\mathrm{CH}), 126.4,126.8$ (2 x ArCH), 130.1 (Ar-C), 141.5, 142.1 ( 2 x Ar-CH), 144.6 (Ar-C). LC-MS: Calcd for double quaternary cation $\mathrm{C}_{22} \mathrm{H}_{26} \mathrm{~N}_{4}: \mathrm{M}^{2+}$ 173.11, [M-H] ${ }^{+}$345.21; found: $\mathrm{M}^{2+}$ 173.04, [M-H] ${ }^{+} 345.64$.

$\boldsymbol{N}^{\mathbf{1}}$ - and $\boldsymbol{N}^{\mathbf{2}}$-(4-Chlorobutyl)benzotriazole. Prepared according to method $\mathrm{B}$, using benzotriazole (6.00 g, $50.42 \mathrm{mmol})$ 1,3-dichlorobutane $(150 \mathrm{~mL})$, tetrabutylammonium iodide (0.30 g, 5\% w/w), KOH (16.96 g, $0.30 \mathrm{~mol})$ and $\mathrm{K}_{2} \mathrm{CO}_{3}(17.40 \mathrm{~g}, 0.13 \mathrm{~mol})$. Work up gave the title compounds. $N^{1}$-(4-Chlorobutyl)benzotriazole: brown crystals (2.56 g, 24\%), m.p. 104-106 ${ }^{\circ} \mathrm{C}$, IR $\left(v_{\max }, \mathrm{NaCl} / \mathrm{cm}^{-1}\right)$ 3051, 2958, 1566, 1445, 1327, 1264. ${ }^{1} \mathrm{H}$ NMR (400 MHz, $\left.\mathrm{CDCl}_{3}\right), \delta_{\mathrm{H}}$ 1.85, 2.17 (each 2H, m, $\left.\mathrm{CH}_{2}\right) 3.56\left(2 \mathrm{H}, \mathrm{t}, J=6.4 \mathrm{~Hz}, \mathrm{CH}_{2} \mathrm{Cl}\right), 4.68\left(2 \mathrm{H}, \mathrm{t}, J=6.9 \mathrm{~Hz}, \mathrm{CH}_{2} \mathrm{~N}\right)$, $7.38(1 \mathrm{H}, \mathrm{d}, J=7.1 \mathrm{~Hz}, \mathrm{Ar}-\mathrm{H}), 7.51(2 \mathrm{H}, \mathrm{m}, \mathrm{Ar}-\mathrm{H}), 8.05(1 \mathrm{H}, \mathrm{d}, J=8.4 \mathrm{~Hz}, \mathrm{Ar}-\mathrm{H}) .{ }^{13} \mathrm{C}$ NMR (100 MHz, $\left.\mathrm{CDCl}_{3}\right), \delta_{\mathrm{C}}$ 26.8, $29.4\left(2 \times \mathrm{CH}_{2}\right), 44.1\left(\mathrm{CH}_{2} \mathrm{I}\right), 47.3\left(\mathrm{CH}_{2} \mathrm{~N}\right), 109.2,120.1,123.9$, 127.4 (4 x Ar-CH), 132.8, 146.0 (2 x Ar-C). MS, $m / z=210\left(\mathrm{MH}^{+}\right), 175,137,121$. HRMS: Calcd for $\mathrm{C}_{10} \mathrm{H}_{12}{ }^{35} \mathrm{ClN}_{3}$ : $\mathrm{MH}^{+}$210.0792; found: $\mathrm{MH}^{+}$210.0794. $\mathrm{N}^{2}$-(4-Chlorobutyl)benzotriazole: brown crystals $(2.23 \mathrm{~g}, 21 \%)$, m.p. $87-88{ }^{\circ} \mathrm{C}$, IR $\left(v_{\max }, \mathrm{NaCl} / \mathrm{cm}^{-1}\right) 2980,1699,1504,1385$, 1240. ${ }^{1} \mathrm{H}$ NMR (400 MHz, $\mathrm{CDCl}_{3}$ ), $\delta_{\mathrm{H}} 1.81,2.28$ (each $2 \mathrm{H}, \mathrm{m}, \mathrm{CH}_{2}$ ), $3.55(2 \mathrm{H}, \mathrm{t}, \mathrm{J}=6.4 \mathrm{~Hz}$, $\left.\mathrm{CH}_{2} \mathrm{I}\right), 4.76\left(2 \mathrm{H}, \mathrm{t}, J=6.8 \mathrm{~Hz}, \mathrm{CH}_{2} \mathrm{~N}\right), 7.36,7.87$ (each $\left.2 \mathrm{H}, \mathrm{m}, \mathrm{Ar}-\mathrm{H}\right) .{ }^{13} \mathrm{C} \mathrm{NMR}(100 \mathrm{MHz}$, $\left.\mathrm{CDCl}_{3}\right), \delta_{\mathrm{C}} 27.1,29.3$, (2 x CH$), 44.1\left(\mathrm{CH}_{2} \mathrm{I}\right), 55.6\left(\mathrm{CH}_{2} \mathrm{~N}\right), 118.0,126.3(2 \mathrm{x} \mathrm{Ar}-\mathrm{CH}), 144.3$ $\left(\right.$ Ar-C). MS, $m / z=210\left(\mathrm{MH}^{+}\right), 175,137,121$. HRMS: Calcd for $\mathrm{C}_{10} \mathrm{H}_{12}{ }^{35} \mathrm{ClN}_{3}$ requires: $\mathrm{MH}^{+}$ 210.0792; Found: $\mathrm{MH}^{+} 210.0791$.

$\boldsymbol{N}^{\mathbf{1}}$-(4-Iodobutyl)benzotriazole (11e). Prepared according to general procedure C, using $N^{1}$-(4chlorobutyl)benzotriazole $(2.00 \mathrm{~g}, 9.55 \mathrm{mmol})$ and dry $\mathrm{NaI}(7.15 \mathrm{~g}, 47.67 \mathrm{mmol})$ in dry acetone $(100 \mathrm{~mL})$. Work up gave the title compound as a pale yellow oil $(2.01 \mathrm{~g}, 70 \%)$, IR ( $v_{\max }$, 
$\mathrm{NaCl} / \mathrm{cm}^{-1}$ ) 3030, 2934, 1601, 1462. ${ }^{1} \mathrm{H}$ NMR (400 MHz, $\mathrm{CDCl}_{3}$ ), $\delta_{\mathrm{H}}$ 1.83, 2.13 (each 2H, m, $\left.\mathrm{CH}_{2}\right), 3.18\left(2 \mathrm{H}, \mathrm{t}, J=6.7 \mathrm{~Hz}, \mathrm{CH}_{2} \mathrm{I}\right), 4.68\left(2 \mathrm{H}, \mathrm{t}, J=6.9 \mathrm{~Hz}, \mathrm{CH}_{2} \mathrm{~N}\right), 7.33(1 \mathrm{H}, \mathrm{d}, J=6.2 \mathrm{~Hz}$, Ar$\mathrm{H}), 7.48(2 \mathrm{H}, \mathrm{m}, \mathrm{Ar}-\mathrm{H}), 8.06(1 \mathrm{H}, \mathrm{d}, J=7.2 \mathrm{~Hz}, \mathrm{Ar}-\mathrm{H}) .{ }^{13} \mathrm{C} \mathrm{NMR}\left(100 \mathrm{MHz}, \mathrm{CDCl}_{3}\right), \delta_{\mathrm{C}} 6.9$ $\left(\mathrm{CH}_{2} \mathrm{I}\right), 32.5,35.4$ (2 x CH$), 55.5\left(\mathrm{CH}_{2} \mathrm{~N}\right), 119.2,123.8,124.1,128.9(4 \times \mathrm{Ar}-\mathrm{CH}), 137.0,142.7$ ( 2 x ArC). MS, $m / z=310\left(M^{+}\right)$, 174, 146, 120. HRMS: Calcd for $\mathrm{C}_{10} \mathrm{H}_{12} \mathrm{IN}_{3}: \mathrm{M}^{+} 301.0078$; found: $\mathrm{M}^{+}$301.0073. Anal. Calcd for $\mathrm{C}_{10} \mathrm{H}_{12} \mathrm{IN}_{3}$ : C, 39.89; H, 4.02; N, 13.95\%; Found: C, 39.52; H, 4.00; N, 13.63\%.

$N^{2}$-(4-Iodobutyl)benzotriazole (11f). Prepared according to general procedure C, using $N^{2}$-(4chlorobutyl)benzimidazole (2.00 g, $9.55 \mathrm{mmol})$ and dry $\mathrm{NaI}(7.15 \mathrm{~g}, 47.67 \mathrm{mmol})$ in dry acetone $(100 \mathrm{~mL})$. Work up gave the title compound as a pale yellow solid $(2.00 \mathrm{~g}, 70 \%)$, $\left(v_{\max }\right.$, $\mathrm{NaCl} / \mathrm{cm}^{-1}$ ) 3101, 2944, 1599, 1445, 1221. ${ }^{1} \mathrm{H}$ NMR (400 MHz, $\left.\mathrm{CDCl}_{3}\right), \delta_{\mathrm{H}} 1.84$ (2H, tt, $J=6.9$, $\left.7.3 \mathrm{~Hz}, \mathrm{CH}_{2}\right), 2.16\left(2 \mathrm{H}, \mathrm{dt}, J=6.8,7.3 \mathrm{~Hz}, \mathrm{CH}_{2}\right), 3.22\left(2 \mathrm{H}, \mathrm{t}, J=6.9 \mathrm{~Hz}, \mathrm{CH}_{2} \mathrm{I}\right), 4.77(2 \mathrm{H}, \mathrm{t}, J=$ $6.8 \mathrm{~Hz}, \mathrm{CH}_{2} \mathrm{~N}$ ), 7.36, 7.48 (each $\left.2 \mathrm{H}, \mathrm{m}, \mathrm{Ar}-\mathrm{H}\right) .{ }^{13} \mathrm{C} \mathrm{NMR}\left(100 \mathrm{MHz}, \mathrm{CDCl}_{3}\right), \delta_{\mathrm{C}} 5.8\left(\mathrm{CH}_{2} \mathrm{I}\right)$, 30.1, $30.8\left(2 \times \mathrm{CH}_{2}\right), 55.3\left(\mathrm{CH}_{2} \mathrm{~N}\right), 118.0,126.4$ (2 x Ar-CH), 144.3 (Ar-C). MS, m/z = $301\left(\mathrm{M}^{+}\right)$, 174, 147, 120. HRMS: Calcd for $\mathrm{C}_{10} \mathrm{H}_{12} \mathrm{IN}_{3}: \mathrm{M}^{+}$301.0078; found: $\mathrm{M}^{+}$301.0069. Anal. Calcd for $\mathrm{C}_{10} \mathrm{H}_{12} \mathrm{IN}_{3}$ : C, 39.89; H, 4.02; N, 13.95\%; Found: C, 39.33; H, 4.14; N, 14.28\%.

$\boldsymbol{N}$-tert-butoxycarbonyl-(R)-cysteine ethyl ester (5). To a dry round-bottomed flask was added (R)-cysteine ethyl ester hydrochloride (10.00 g, $53.86 \mathrm{mmol})$, di-tert-butyl dicarbonate (11.76 g, $53.94 \mathrm{mmol})$ and diisopropylethylamine $(11.18 \mathrm{~mL}, 8.30 \mathrm{~g}, 64.31 \mathrm{mmol})$ in dry $\mathrm{CH}_{2} \mathrm{Cl}_{2}(200$ $\mathrm{mL}$ ). The mixture was stirred for $2 \mathrm{~h}$ at $20^{\circ} \mathrm{C}$ and then washed with hydrochloric acid (1M; $2 \mathrm{x}$ $100 \mathrm{~mL}), \mathrm{NaHCO}_{3}$ solution (1M; 2 x $\left.100 \mathrm{ml}\right)$, and water $(2 \times 100 \mathrm{~mL})$, dried $\left(\mathrm{MgSO}_{4}\right)$, and the solvent removed under reduced pressure. Work up gave the title compound as off white oil (12.62 g, 94\%), ( $v_{\max }\left(\mathrm{NaCl} / \mathrm{cm}^{-1}\right)$ 3367, 2979, 1712, 1507, 1368, 1165, 1029. ${ }^{1} \mathrm{H}$ NMR (400 $\left.\mathrm{MHz}, \mathrm{CDCl}_{3}\right), \delta_{\mathrm{H}} 1.22\left(3 \mathrm{H}, \mathrm{t}, J=7.1 \mathrm{~Hz}, \mathrm{CH}_{2} \mathrm{CH}_{3}\right), 1.38\left(9 \mathrm{H}, \mathrm{s}, \mathrm{C}\left(\mathrm{CH}_{3}\right)_{3}\right), 2.90\left(2 \mathrm{H}, \mathrm{m}, \mathrm{CH}_{2} \mathrm{~S}\right)$, $4.17\left(2 \mathrm{H}, \mathrm{q}, J=7.1 \mathrm{~Hz}, \mathrm{CH}_{2} \mathrm{CH}_{3}\right), 4.50(1 \mathrm{H}, \mathrm{m}, \mathrm{CH}), 5.43(1 \mathrm{H}, \mathrm{br}, \mathrm{NH}) .{ }^{13} \mathrm{C} \mathrm{NMR}(100 \mathrm{MHz}$,

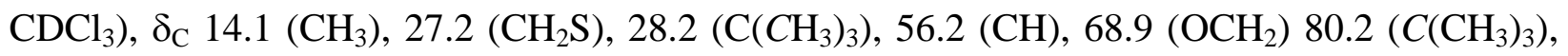
$170.4(\mathrm{C}=\mathrm{O})$. MS, $\mathrm{m} / \mathrm{z}=250\left(\mathrm{MH}^{+}\right), 211,193$, 135. HRMS: Calcd for $\mathrm{C}_{10} \mathrm{H}_{19} \mathrm{NO}_{4} \mathrm{~S}: \mathrm{MH}^{+}$ 250.1107; found: $\mathrm{MH}^{+} 250.1108$.

General procedure for preparation of S-linked heterocyclic amino acids, exemplified by $N$ tert-butoxycarbonyl-S-[2-(3-benzoyluracil-1-yl)ethyl]cysteine ethyl ester (13a)

To a flame-dried round-bottom flask under a positive pressure of nitrogen was added NaH (60\% in mineral oil; $0.10 \mathrm{~g}, 2.50 \mathrm{mmol}$,), which was washed for $5 \mathrm{~min}$ with hexane, the hexane decanted, and then cooled to $0{ }^{\circ} \mathrm{C}$. Dry THF $(25 \mathrm{~mL})$ was added to the reaction vessel followed by $N$-tert-butoxycarbonyl-(R)-cysteine ethyl ester $5(0.50 \mathrm{~g}, 2.01 \mathrm{mmol})$ in dry THF (25 mL). The reaction mixture was allowed to warm to $20{ }^{\circ} \mathrm{C}$ over $15 \mathrm{~min}$ before the addition of 3benzoyl-1-(2-iodoethyl)uracil 9a (2.98 g, $8.05 \mathrm{mmol}$ ), after which the reaction was heated at reflux for $16 \mathrm{~h}$. The THF was then removed under reduced pressure and the residue taken up in $\mathrm{CH}_{2} \mathrm{Cl}_{2}$-water $(100 \mathrm{~mL}, 1: 1 \mathrm{v} / \mathrm{v})$. The $\mathrm{CH}_{2} \mathrm{Cl}_{2}$ layer was separated, washed with water $(20 \mathrm{~mL})$, 
dried $\left(\mathrm{MgSO}_{4}\right)$ and the solvent removed under reduced pressure. The residue was purified by column chromatography, eluting with ethyl acetate:hexane, to afford the title compound as a yellow oil (0.62 g, 64\%), IR ( $\left.v_{\max }, \mathrm{NaCl} / \mathrm{cm}^{-1}\right)$ 3416, 2930 2857, 2357, 1713, 1650, 1555, 1453, 1249, 1162. ${ }^{1} \mathrm{H}$ NMR (400 MHz, $\left.\mathrm{CDCl}_{3}\right), \delta_{\mathrm{H}} 1.19\left(3 \mathrm{H}, \mathrm{t}, J=7.1 \mathrm{~Hz}, \mathrm{CH}_{2} \mathrm{CH}_{3}\right), 1.95(9 \mathrm{H}, \mathrm{s}$, $\left.\mathrm{C}\left(\mathrm{CH}_{3}\right)_{3}\right), 3.13\left(1 \mathrm{H}\right.$, apparent t, $\left.J=6.6 \mathrm{~Hz}, \mathrm{CH}_{2} \mathrm{~S}\right), 3.35\left(2 \mathrm{H}, \mathrm{t}, J=6.4 \mathrm{~Hz}, \mathrm{CH}_{2} \mathrm{~S}\right), 4.04$ (2H, q, $J$ $\left.=7.1 \mathrm{~Hz}, \mathrm{CH}_{2} \mathrm{CH}_{3}\right), 4.86\left(3 \mathrm{H}, \mathrm{m}, \mathrm{CH}_{2} \mathrm{~N}, \mathrm{CH}\right), 5.72(1 \mathrm{H}, \mathrm{d}, J=8.0 \mathrm{~Hz}, \mathrm{COCH}=\mathrm{CH}), 7.19-7.62$ $(6 \mathrm{H}, \mathrm{m}, \mathrm{COCH}=\mathrm{CH}, \mathrm{Ar}-\mathrm{H},) .{ }^{13} \mathrm{C} \mathrm{NMR}\left(100 \mathrm{MHz}, \mathrm{CDCl}_{3}\right), \delta_{\mathrm{C}} 7.1\left(\mathrm{CH}_{2} \mathrm{CH}_{3}\right), 20.7\left(\mathrm{C}\left(\mathrm{CH}_{3}\right)_{3}\right)$, 50.0, $52.4\left(2 \times \mathrm{CH}_{2} \mathrm{~S}\right), 59.2\left(\mathrm{CH}_{2} \mathrm{~N}\right), 62.1\left(\mathrm{OCH}_{2}\right), 64.9\left(\mathrm{C}\left(\mathrm{CH}_{3}\right)_{3}\right), 68.3(\mathrm{CH}), 100.5$ $(\mathrm{COCH}=\mathrm{CH}), 127.2$ (2 x Ar-CH), $128.0(\mathrm{COCH}=\mathrm{CH}), 130.5$ (Ar-CH), 143.7 (Ar-C), 148.3, 155.4, 161.2, 167.4, 169.9 (5 x C=O). MS, $m / z=272\left(\mathrm{MH}^{+}-\mathrm{NHBoc}, \mathrm{COPh}\right), 243$, 188. Anal. Calcd for $\mathrm{C}_{23} \mathrm{H}_{29} \mathrm{~N}_{3} \mathrm{O}_{7} \mathrm{~S}$ : C, 56.20; H, 5.95; N, 8.55\%: Found C, 56.77; H, 5.82; N, 8.30\%.

$\mathbf{N}$-tert-Butoxycarbonyl-S-[2-(3-benzoylthymin-1-yl)ethyl]cysteine ethyl ester (13b). Prepared according to the method used for 13a but allowing the mixture to warm to $20{ }^{\circ} \mathrm{C}$ over $20 \mathrm{~min}$ before the addition of 3-benzoyl-1-(2-iodoethyl)thymine 9b (3.09 g, $8.05 \mathrm{mmol})$. The title compound was isolated as a yellow oil (0.61 g, 62\%), IR ( $\left.v_{\max }, \mathrm{NaCl} / \mathrm{cm}^{-1}\right) 3284,2978,2930$, 1745, 1698, 1657, 1454, 1367, 1251, 1175. ${ }^{1} \mathrm{H}$ NMR (400 MHz, $\left.\mathrm{CDCl}_{3}\right), \delta_{\mathrm{H}} 1.19$ (3H, t, $J=6.6$ $\left.\mathrm{Hz}, \mathrm{CH}_{2} \mathrm{CH}_{3}\right), 1.75\left(9 \mathrm{H}, \mathrm{s}, \mathrm{C}\left(\mathrm{CH}_{3}\right)_{3}\right), 1.81\left(3 \mathrm{H}, \mathrm{s}, \mathrm{CH}_{3}\right), 3.26\left(1 \mathrm{H}\right.$, apparent t, $\left.J=7.8 \mathrm{~Hz}, \mathrm{CH}_{2} \mathrm{~S}\right)$, $3.39\left(2 \mathrm{H}, \mathrm{t}, J=7.1 \mathrm{~Hz}, \mathrm{CH}_{2} \mathrm{~S}\right), 4.11\left(2 \mathrm{H}, \mathrm{q}, J=6.6 \mathrm{~Hz}, \mathrm{CH}_{2} \mathrm{CH}_{3}\right), 4.16(2 \mathrm{H}, \mathrm{t}, J=7.1 \mathrm{~Hz}$, $\left.\mathrm{CH}_{2} \mathrm{~N}\right), 4.68(1 \mathrm{H}, \mathrm{t}, J=7.8 \mathrm{~Hz}, \mathrm{CH}), 5.71(1 \mathrm{H}, \mathrm{s}, \mathrm{C}=\mathrm{CH}), 7.24-7.95(5 \mathrm{H}, \mathrm{m}, \mathrm{Ar}-\mathrm{H}) .{ }^{13} \mathrm{C}$ NMR (100 MHz, $\left.\mathrm{CDCl}_{3}\right), \delta_{\mathrm{C}} 11.2\left(\mathrm{CH}_{2} \mathrm{CH}_{3}\right), 11.7\left(\mathrm{CCH}_{3}\right) 27.3\left(\mathrm{C}\left(\mathrm{CH}_{3}\right)_{3}\right), 40.9$, $48.3\left(2 \times \mathrm{CH}_{2} \mathrm{~S}\right), 54.0$ $\left(C\left(\mathrm{CH}_{3}\right)_{3}\right), 56.8\left(\mathrm{CH}_{2} \mathrm{~N}\right), 65.9\left(\mathrm{OCH}_{2}\right), 99.0(\mathrm{CH}), 106.5\left(\mathrm{CH}_{3} \mathrm{C}=\mathrm{CH}\right), 107.6(\mathrm{Ar}-\mathrm{CH}), 135.3$ $\left(\mathrm{CCH}_{3}=\mathrm{CH}\right), 135.7,140.3$ (2 x Ar-CH), 150.1 (Ar-C), 160.0, 162.6, 163.1, 163.2, 163.4 (5 x $\mathrm{C}=\mathrm{O})$. MS, $\mathrm{m} / \mathrm{z}=388\left(\mathrm{M}^{+}-\mathrm{H}_{2} \mathrm{NBoc}\right), 185,127$. Anal. Calcd for $\mathrm{C}_{24} \mathrm{H}_{31} \mathrm{~N}_{3} \mathrm{O}$ - S: C, 57.02; $\mathrm{H}, 6.18$; N, 8.31\%. Found: C, 56.81; H, 6.40; N, 8.52\%.

$\mathrm{N}$-tert-Butoxycarbonyl-S-[2-(indol-1-yl)ethyl]cysteine ethyl ester (13c). Prepared according to the method used for 13a but allowing the mixture to warm to $20{ }^{\circ} \mathrm{C}$ over $10 \mathrm{~min}$ before the addition of $\mathrm{N}$-(2-iodoethyl)indole $9 \mathrm{c}(2.17 \mathrm{~g}, 8.01 \mathrm{mmol})$, and heating at reflux for $48 \mathrm{~h}$. The title compound was isolated as a yellow oil $(0.24 \mathrm{~g}, 31 \%) .{ }^{1} \mathrm{H} \mathrm{NMR}\left(400 \mathrm{MHz}, \mathrm{CDCl}_{3}\right), \delta_{\mathrm{H}} 1.23(3 \mathrm{H}$, t, $\left.J=7.1 \mathrm{~Hz}, \mathrm{CH}_{2} \mathrm{CH}_{3}\right), 1.36\left(9 \mathrm{H}, \mathrm{s}, \mathrm{C}\left(\mathrm{CH}_{3}\right)_{3}\right), 1.73,2.92$ (each $\left.2 \mathrm{H}, \mathrm{m}, \mathrm{CH}_{2} \mathrm{~S}\right), 4.12(2 \mathrm{H}, \mathrm{m}$, $\left.\mathrm{CH}_{2} \mathrm{~N}\right), 4.14$ (2H, q, $\left.J=7.1 \mathrm{~Hz}, \mathrm{CH}_{2} \mathrm{CH}_{3}\right), 4.49$ (1 H, m, CH), 5.29 (1H, br, NH), 7.09-7.26, 7.37-7.51, 7.58-7.63 (each 2H, m, Ar-H). ${ }^{13} \mathrm{C}$ NMR (100 MHz, $\left.\mathrm{CDCl}_{3}\right), \delta_{\mathrm{C}} 14.1\left(\mathrm{CH}_{2} \mathrm{CH}_{3}\right), 28.3$ $\left(\mathrm{C}\left(\mathrm{CH}_{3}\right)_{3}\right), 29.7\left(\mathrm{CH}_{2} \mathrm{~S}\right), 30.3\left(\mathrm{C}\left(\mathrm{CH}_{3}\right)_{3}\right), 31.9\left(\mathrm{CH}_{2} \mathrm{~S}\right), 45.1\left(\mathrm{CH}_{2} \mathrm{~N}\right), 61.8\left(\mathrm{OCH}_{2}\right), 107.8(\mathrm{CH})$, 126.4 (Ar-C), 128.4, 128.6, 129.2, 131.9, 132.2, 132.9 (6 x Ar-CH), 143.1 (Ar-C), 157.6, 179.9 (2 x C=O). MS, $m / z=347\left(\mathrm{M}^{+}-\mathrm{OC}_{2} \mathrm{H}_{5}\right), 291,249$, 232, 203, 176, 146. HRMS: Calcd for $\mathrm{C}_{20} \mathrm{H}_{28} \mathrm{~N}_{2} \mathrm{O}_{4} \mathrm{~S}$ : [M-OC $\left.\mathrm{H}_{5}\right]^{+}$347.1429; found: [M-OC $\left.{ }_{2} \mathrm{H}_{5}\right]^{+} 347.1431$.

N-tert-Butoxycarbonyl-S-[2-(benzimidazol-1-yl)ethyl]cysteine ethyl ester (13d). Prepared according to the method used for 13a but allowing the mixture to warm to $20{ }^{\circ} \mathrm{C}$ over $20 \mathrm{~min}$ before the addition of $N^{1}$-(2-iodoethyl)benzimidazole $9 \mathrm{~d}$ (2.18 g, $8.01 \mathrm{mmol}$ ). The title compound was isolated as a yellow oil $(0.44 \mathrm{~g}, 66 \%)$, IR $\left(v_{\max }, \mathrm{NaCl} / \mathrm{cm}^{-1}\right)$ 3352, 3223, 2976, 1708, 1494, 1458, 1366, 1250, 1165. ${ }^{1} \mathrm{H}$ NMR (400 MHz, $\left.\mathrm{CDCl}_{3}\right), \delta_{\mathrm{H}} 1.18(3 \mathrm{H}, \mathrm{t}, J=7.2 \mathrm{~Hz}$, 
$\left.\mathrm{CH}_{2} \mathrm{CH}_{3}\right), 1.36$ (9H, s, C( $\left.\left(\mathrm{CH}_{3}\right)_{3}\right), 2.88\left(2 \mathrm{H}, 2 \mathrm{x} \mathrm{dd}, J=6.3,6.6 \mathrm{~Hz}, \mathrm{CH}_{2} \mathrm{~S}\right), 2.91(2 \mathrm{H}, \mathrm{t}, J=7.0$ $\left.\mathrm{Hz}, \mathrm{SCH}_{2}\right), 4.11\left(2 \mathrm{H}, \mathrm{q}, J=7.2 \mathrm{~Hz}, \mathrm{CH}_{2} \mathrm{CH}_{3}\right), 4.29\left(2 \mathrm{H}, \mathrm{t}, J=7.0 \mathrm{~Hz}, \mathrm{CH}_{2} \mathrm{~N}\right), 4.41(1 \mathrm{H}, \mathrm{m}, \mathrm{CH})$, $5.23(1 \mathrm{H}, \mathrm{br}, \mathrm{NH}), 7.20-7.75(4 \mathrm{H}, \mathrm{m}, \mathrm{Ar}-\mathrm{H}), 7.88(1 \mathrm{H}, \mathrm{s}, \mathrm{CH}=\mathrm{N}) .{ }^{13} \mathrm{C} \mathrm{NMR}\left(100 \mathrm{MHz}, \mathrm{CDCl}_{3}\right)$, $\delta_{\mathrm{C}} 14.2\left(\mathrm{CH}_{2} \mathrm{CH}_{3}\right), 28.3\left(\mathrm{C}\left(\mathrm{CH}_{3}\right)_{3}\right), 32.3,34.8\left(2 \times \mathrm{CH}_{2} \mathrm{~S}\right), 42.9\left(\mathrm{C}\left(\mathrm{CH}_{3}\right)_{3}\right), 44.9\left(\mathrm{CH}_{2} \mathrm{~N}\right), 61.9$ $\left(\mathrm{OCH}_{2}\right), 109.4(\mathrm{CH}), 120.5,122.4,123.1,128.6,132.1$ (5 x Ar-CH), 133.3, 143.7 (2 x Ar-C), 155.2, 170.7 ( 2 x C=O). MS, $\mathrm{m} / \mathrm{z}=394\left(\mathrm{M}^{+}\right)$, 338, 279, 220, 192, 160, 146, 118. HRMS: Calcd for $\mathrm{C}_{19} \mathrm{H}_{27} \mathrm{~N}_{3} \mathrm{O}_{4} \mathrm{~S}$ : $\mathrm{MH}^{+}$394.1795; found: $\mathrm{MH}^{+}$394.1802. Anal. Calcd for $\mathrm{C}_{19} \mathrm{H}_{27} \mathrm{~N}_{3} \mathrm{O}_{4} \mathrm{~S}$ : C, 57.99; H, 6.92; N, 10.68\%; Found C, 58.22; H, 6.80; N, 10.39\%.

$\boldsymbol{N}$-tert-Butoxycarbonyl-S-[2-(benzotriazol-1-yl)ethyl]cysteine ethyl ester (13e). Prepared according to the method used for 13a but using $\mathrm{NaH}$ (60\% in mineral oil; $0.04 \mathrm{~g}, 1.00 \mathrm{mmol}$ ), and $N$-tert-butoxycarbonyl-(R)-cysteine ethyl ester $5(0.20 \mathrm{~g}, 0.80 \mathrm{mmol})$, and allowing the mixture to warm to $20^{\circ} \mathrm{C}$ over $10 \mathrm{~min}$ before the addition of $N^{1}$-(2-iodoethyl)benzotriazole 9e (0.88 g, $3.22 \mathrm{mmol})$. The title compound was isolated as a yellow oil $(0.25 \mathrm{~g}, 75 \%)$, IR ( $v_{\max }$, $\mathrm{NaCl} / \mathrm{cm}^{-1}$ ) 3444, 2915, 2065, 1634, 1495, 1393, 1247, 1162. ${ }^{1} \mathrm{H}$ NMR (400 MHz, $\left.\mathrm{CDCl}_{3}\right), \delta_{\mathrm{H}}$ $1.24\left(3 \mathrm{H}, \mathrm{t}, J=7.0 \mathrm{~Hz}, \mathrm{CH}_{2} \mathrm{CH}_{3}\right), 1.60\left(9 \mathrm{H}, \mathrm{s}, \mathrm{C}\left(\mathrm{CH}_{3}\right)_{3}\right), 3.09\left(2 \mathrm{H}\right.$, br t, $\left.J=6.9 \mathrm{~Hz}, \mathrm{CH}_{2} \mathrm{~S}\right), 3.22$ (2H, apparent t, $\left.J=6.7 \mathrm{~Hz}, \mathrm{CH}_{2} \mathrm{~S}\right), 4.08\left(2 \mathrm{H}, \mathrm{q}, J=7.0 \mathrm{~Hz}, \mathrm{CH}_{2} \mathrm{CH}_{3}\right), 4.74(2 \mathrm{H}, \mathrm{t}, J=6.9 \mathrm{~Hz}$, $\left.\mathrm{CH}_{2} \mathrm{~N}\right), 4.84(1 \mathrm{H}, \mathrm{t}, J=6.7 \mathrm{~Hz}, \mathrm{CH}), 5.23(1 \mathrm{H}, \mathrm{br}, \mathrm{NH}), 7.26-7.48$ (3H, m, Ar-H), 7.98 (1H, m, Ar-H). ${ }^{13} \mathrm{C}$ NMR (100 MHz, $\left.\mathrm{CDCl}_{3}\right), \delta_{\mathrm{C}} 12.3\left(\mathrm{CH}_{2} \mathrm{CH}_{3}\right), 29.9\left(\mathrm{C}\left(\mathrm{CH}_{3}\right)_{3}\right), 33.2,35.2\left(2 \times \mathrm{CH}_{2} \mathrm{~S}\right)$, $54.0\left(\mathrm{CH}_{2} \mathrm{~N}\right), 58.5\left(\mathrm{OCH}_{2}\right), 64.0\left(\mathrm{C}\left(\mathrm{CH}_{3}\right)_{3}\right), 111.9(\mathrm{CH}), 128.6,130.6,131.1,139.1(4 \mathrm{x} \mathrm{Ar}-\mathrm{CH})$, 142.1, 146.9 (2 x Ar-C), 170.2, 184.6 (2 x C=O). MS, $m / z=395\left(\mathrm{MH}^{+}\right), 360,338,294,250,191$, 176. HRMS: Calcd for $\mathrm{C}_{18} \mathrm{H}_{26} \mathrm{~N}_{4} \mathrm{O}_{4} \mathrm{~S}: \mathrm{MH}^{+}$395.1747; found: $\mathrm{MH}^{+} 395.1750$.

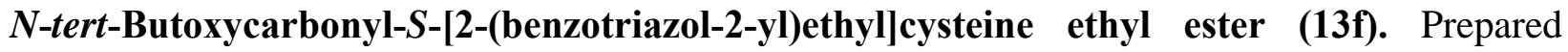
according to the method used for 13a but using $\mathrm{NaH}$ (60\% in mineral oil; $0.04 \mathrm{~g}, 1.00 \mathrm{mmol}$ ) and $N$-tert-butoxycarbonyl- $(R)$-cysteine ethyl ester $5(0.20 \mathrm{~g}, 0.80 \mathrm{mmol})$, and allowing the mixture to warm to $20{ }^{\circ} \mathrm{C}$ over $10 \mathrm{~min}$ before the addition of $N^{2}$-(2-iodoethyl)benzotriazole $9 \mathrm{f}(0.88 \mathrm{~g}$, $3.22 \mathrm{mmol})$. The title compound was isolated as a yellow oil $(0.25 \mathrm{~g}, 74 \%), \mathrm{IR}\left(v_{\max }, \mathrm{NaCl} / \mathrm{cm}^{-1}\right)$ 3368, 2976, 1717, 1688, 1506, 1455, 1165, 1128. ${ }^{1} \mathrm{H}$ NMR (400 MHz, $\left.\mathrm{CDCl}_{3}\right), \delta_{\mathrm{H}} 1.29$ (3H, t, $J$ = 7.1 Hz, $\left.\mathrm{CH}_{2} \mathrm{CH}_{3}\right), 1.47\left(9 \mathrm{H}, \mathrm{s}, \mathrm{C}\left(\mathrm{CH}_{3}\right)_{3}\right), 3.00\left(2 \mathrm{H}, \mathrm{t}, J=6.9 \mathrm{~Hz}, \mathrm{CH}_{2} \mathrm{~S}\right), 3.29(2 \mathrm{H}, \mathrm{t}, J=7.1$ $\left.\mathrm{Hz}, \mathrm{CH}_{2} \mathrm{~S}\right), 4.23\left(2 \mathrm{H}, \mathrm{q}, J=7.1 \mathrm{~Hz}, \mathrm{CH}_{2} \mathrm{CH}_{3}\right), 4.55(1 \mathrm{H}, \mathrm{m}, \mathrm{CH}), 4.93\left(2 \mathrm{H}, \mathrm{t}, J=6.9 \mathrm{~Hz}, \mathrm{CH}_{2} \mathrm{~N}\right)$, $5.44(1 \mathrm{H}$, br, $\mathrm{NH}), 7.40,7.88$ (each $2 \mathrm{H}, \mathrm{m}, \mathrm{Ar}-\mathrm{H}) .{ }^{13} \mathrm{C} \mathrm{NMR}\left(100 \mathrm{MHz}, \mathrm{CDCl}_{3}\right), \delta_{\mathrm{C}} 14.1$

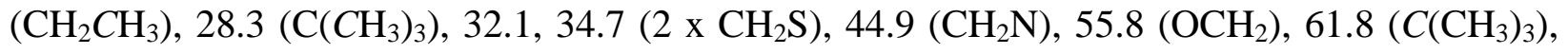
118.0 (CH), 126.5, 133.3 (2 x Ar-CH), 144.4 (Ar-C), 177.9, 180.2 (2 x C=O). MS, m/z = 395 $\left(\mathrm{MH}^{+}\right)$, 360, 338, 294, 250, 191, 176. HRMS: Calcd for $\mathrm{C}_{18} \mathrm{H}_{26} \mathrm{~N}_{4} \mathrm{O}_{4} \mathrm{~S}: \mathrm{MH}^{+}$395.1747; found: $\mathrm{MH}^{+}$395.1751.

N-tert-Butoxycarbonyl-S-[2-(6-tert-butyloxycarbonylaminopurin-9-yl)ethyl]cysteine ethyl ester (13g). Prepared according to the method used for 13a but allowing the mixture to warm to $20{ }^{\circ} \mathrm{C}$ over $10 \mathrm{~min}$ before the addition of $N^{9}$-(2-Iodoethyl)-6-tert-butoxycarbonylaminopurine $\mathbf{9 g}$ (3.12 g, $8.02 \mathrm{mmol})$. The title compound was isolated as a yellow oil (0.42 g, 41\%), IR ( $v_{\max }$, $\mathrm{NaCl} / \mathrm{cm}^{-1}$ ) 3419, 2979, 1738, 1701, 1657, 1455, 1367, 1252, 1175. ${ }^{1} \mathrm{H}$ NMR (250 MHz, $\left.\mathrm{CDCl}_{3}\right)$, $\delta_{\mathrm{H}} 1.14\left(3 \mathrm{H}, \mathrm{t}, J=7.2 \mathrm{~Hz}, \mathrm{CH}_{2} \mathrm{CH}_{3}\right), 1.44,1.47$ (each 9H, s, C(CH$\left.)_{3}\right), 3.69-3.81(4 \mathrm{H}, \mathrm{m}, 2 \mathrm{x}$ 
$\mathrm{CH}_{2} \mathrm{~S}$ ), 4.14 (2H, q, J = 7.2 Hz, $\left.\mathrm{CH}_{2} \mathrm{CH}_{3}\right), 4.24\left(2 \mathrm{H}, \mathrm{m}, \mathrm{CH}_{2} \mathrm{~N}\right), 6.00$ (1H, m, CH), 6.48 (2H, br, $\mathrm{NH}$ ), 7.50, 7.58 (each $1 \mathrm{H}, \mathrm{s}, \mathrm{Ar}-\mathrm{H}$ ); this material was not further characterized.

N-tert-Butoxycarbonyl-S-[3-(benzotriazol-1-yl)propyl]cysteine ethyl ester (13h). Prepared according to the method used for 13a but using $\mathrm{NaH}$ (60\% in mineral oil; $0.04 \mathrm{~g}, 1.00 \mathrm{mmol}$ ) and $N$-tert-butoxycarbonyl-(R)-cysteine ethyl ester $5(0.20 \mathrm{~g}, 0.80 \mathrm{mmol})$, and allowing the mixture to warm to $20^{\circ} \mathrm{C}$ over $10 \mathrm{~min}$ before the addition of $N^{1}$-(3-iodopropyl)benzotriazole $10 \mathrm{e}(0.92 \mathrm{~g}$, $3.21 \mathrm{mmol})$. The title compound was isolated as a yellow oil $(0.20 \mathrm{~g}, 59 \%), \mathrm{IR}\left(v_{\max }, \mathrm{NaCl} / \mathrm{cm}^{-1}\right)$ 3447, 2990, 1636, 1556, 1432, 1102. ${ }^{1} \mathrm{H}$ NMR (400 MHz, $\left.\mathrm{CDCl}_{3}\right), \delta_{\mathrm{H}} 1.22\left(3 \mathrm{H}, \mathrm{m}, \mathrm{CH}_{2} \mathrm{CH}_{3}\right)$, $1.43\left(9 \mathrm{H}, \mathrm{s}, \mathrm{C}\left(\mathrm{CH}_{3}\right)_{3}\right), 1.76\left(2 \mathrm{H}, \mathrm{m}, \mathrm{CH}_{2}\right), 2.31,2.74$ (each $\left.2 \mathrm{H}, \mathrm{t}, J=7.0 \mathrm{~Hz}, \mathrm{CH}_{2} \mathrm{~S}\right), 4.22(2 \mathrm{H}$, m, $\left.\mathrm{CH}_{2} \mathrm{CH}_{3}\right), 4.66\left(2 \mathrm{H}, \mathrm{t}, J=7.0 \mathrm{~Hz}, \mathrm{CH}_{2} \mathrm{~N}\right), 5.23(1 \mathrm{H}, \mathrm{m}, \mathrm{CH}), 5.97(1 \mathrm{H}, \mathrm{br}, \mathrm{NH}), 7.31-7.47$ (3H, m, Ar-H), $8.00(1 \mathrm{H}, \mathrm{m}, \mathrm{Ar}-\mathrm{H}) .{ }^{13} \mathrm{C} \mathrm{NMR}\left(100 \mathrm{MHz}, \mathrm{CDCl}_{3}\right), \delta_{\mathrm{C}} 12.5\left(\mathrm{CH}_{3}\right), 16.9\left(\mathrm{CH}_{2}\right)$,

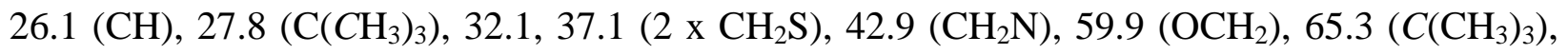
129.5, 130.2 (2 x Ar-CH), 131.3 (Ar-C), 135.4, 143.3 (2 x Ar-CH), 150.0 (Ar-C), 162.9, 170.0 (2 x C=O). MS, $m / z=408\left(M^{+}\right)$, 322, 250, 214, 198, 158, 144, 130, 118. HRMS: Calcd for $\mathrm{C}_{19} \mathrm{H}_{28} \mathrm{~N}_{4} \mathrm{O}_{4} \mathrm{~S}: \mathrm{M}^{+}$408.1831; found: $\mathrm{M}^{+} 408.1832$.

N-tert-Butoxycarbonyl-S-[3-(benzotriazol-2-yl)propyl]cysteine ethyl ester (13i). Prepared according to the method used for 13a but using $\mathrm{NaH}$ (60\% in mineral oil; $0.04 \mathrm{~g}, 1.00 \mathrm{mmol}$ ) and $N$-tert-butoxycarbonyl-(R)-cysteine ethyl ester $5(0.20 \mathrm{~g}, 0.80 \mathrm{mmol})$, and allowing the mixture to warm to $20^{\circ} \mathrm{C}$ over $10 \mathrm{~min}$ before the addition of $N^{2}$-(3-iodopropyl)benzotriazole $10 \mathbf{f}$ (0.92 g, $3.21 \mathrm{mmol})$. The title compound was isolated as a yellow oil $(0.235 \mathrm{~g}, 68 \%), \mathrm{IR}\left(v_{\max }, \mathrm{NaCl} / \mathrm{cm}^{-}\right.$ 1) 3447, 2987, 1653, 1559, 1457, 1372, 1164. ${ }^{1} \mathrm{H}$ NMR (400 MHz, $\left.\mathrm{CDCl}_{3}\right), \delta_{\mathrm{H}} 1.24(3 \mathrm{H}, \mathrm{t}, J=$ $\left.7.1 \mathrm{~Hz}, \mathrm{CH}_{2} \mathrm{CH}_{3}\right), 1.38\left(9 \mathrm{H}, \mathrm{s}, \mathrm{C}\left(\mathrm{CH}_{3}\right)_{3}\right), 1.70\left(2 \mathrm{H}, \mathrm{m}, \mathrm{CH}_{2}\right), 2.50\left(2 \mathrm{H}, \mathrm{t}, J=6.5 \mathrm{~Hz}, \mathrm{CH}_{2} \mathrm{~S}\right), 3.09$ $\left(2 \mathrm{H}, \mathrm{t}, J=6.6 \mathrm{~Hz}, \mathrm{CH}_{2} \mathrm{~S}\right), 4.12\left(2 \mathrm{H}, \mathrm{q}, J=7.1 \mathrm{~Hz}, \mathrm{CH}_{2} \mathrm{CH}_{3}\right), 4.70\left(2 \mathrm{H}, \mathrm{t}, J=6.5 \mathrm{~Hz}, \mathrm{CH}_{2} \mathrm{~N}\right)$, $4.78(1 \mathrm{H}, \mathrm{m}, \mathrm{CH}), 5.00(1 \mathrm{H}, \mathrm{br}, \mathrm{NH}), 7.32,8.00$ (each $2 \mathrm{H}, \mathrm{m}, \mathrm{Ar}-\mathrm{H}) .{ }^{13} \mathrm{C} \mathrm{NMR}(100 \mathrm{MHz}$, $\left.\mathrm{CDCl}_{3}\right), \delta_{\mathrm{C}} 12.3\left(\mathrm{CH}_{3}\right), 25.6\left(\mathrm{C}\left(\mathrm{CH}_{3}\right)_{3}\right), 26.5(\mathrm{CH}), 27.9\left(\mathrm{CH}_{2}\right), 28.5\left(\mathrm{CH}_{2} \mathrm{~N}\right), 31.4\left(\mathrm{CH}_{2} \mathrm{~S}\right), 35.4$ $\left(\mathrm{C}\left(\mathrm{CH}_{3}\right)_{3}\right), 46.2\left(\mathrm{CH}_{2} \mathrm{~S}\right), 54.1\left(\mathrm{OCH}_{2}\right), 118.3$ (Ar-C), 122.3, 125.8 (2 x Ar-CH), 164.8, 177.1 (2 x $\mathrm{C}=\mathrm{O})$. MS, $\mathrm{m} / \mathrm{z}=409\left(\mathrm{MH}^{+}\right), 322,250,214,184,158,144,130,118$. HRMS: Calcd for $\mathrm{C}_{19} \mathrm{H}_{28} \mathrm{~N}_{4} \mathrm{O}_{4} \mathrm{~S}: \mathrm{MH}^{+}$409.1904; found: $\mathrm{MH}^{+} 409.1913$.

N-tert-Butoxycarbonyl-S-[4-(benzotriazol-1-yl)butyl]cysteine ethyl ester (13j). Prepared according to the method used for 13a but using $\mathrm{NaH}$ (60\% in mineral oil; $0.04 \mathrm{~g}, 1.00 \mathrm{mmol}$ ) and $N$-tert-butoxycarbonyl-(R)-cysteine ethyl ester $5(0.20 \mathrm{~g}, 0.80 \mathrm{mmol})$, and allowing the mixture to warm to $20{ }^{\circ} \mathrm{C}$ over $10 \mathrm{~min}$ before the addition of $N^{1}$-(4-iodobutyl)benzotriazole $11 \mathrm{e}(0.97 \mathrm{~g}$, $3.22 \mathrm{mmol})$. The title compound was isolated as a yellow oil $(0.13 \mathrm{~g}, 39 \%), \mathrm{IR}\left(v_{\max }, \mathrm{NaCl} / \mathrm{cm}^{-1}\right)$ 3425, 2928, 1708, 1495, 1367, 1280, 1165. ${ }^{1} \mathrm{H}$ NMR (400 MHz, $\left.\mathrm{CDCl}_{3}\right), \delta_{\mathrm{H}} 1.24$ (3H, t, $J=7.2$ $\mathrm{Hz}, \mathrm{CH}_{2} \mathrm{CH}_{3}$ ), $1.43\left(9 \mathrm{H}, \mathrm{s}, \mathrm{C}\left(\mathrm{CH}_{3}\right)_{3}\right), 1.68,2.17$ (each $\left.2 \mathrm{H}, \mathrm{m}, \mathrm{CH}_{2}\right), 2.58(2 \mathrm{H}, \mathrm{t}, J=7.3 \mathrm{~Hz}$, $\left.\mathrm{CH}_{2} \mathrm{~S}\right), 2.92\left(2 \mathrm{H}, \mathrm{t}, J=4.9 \mathrm{~Hz}, \mathrm{CH}_{2} \mathrm{~S}\right), 4.18\left(2 \mathrm{H}, \mathrm{q}, J=7.2 \mathrm{~Hz} \mathrm{CH}_{2} \mathrm{CH}_{3}\right), 4.67\left(3 \mathrm{H}, \mathrm{m}, \mathrm{CH}_{2} \mathrm{~N}\right.$, $\mathrm{CH}), 5.34$ (1H, br, NH), 7.37-7.53 (2H, m, Ar-H), 7.85, 8.04 (each 1H, m, Ar-H). ${ }^{13} \mathrm{C}$ NMR (100 $\left.\mathrm{MHz}, \mathrm{CDCl}_{3}\right), \delta_{\mathrm{C}} 11.8\left(\mathrm{CH}_{3}\right)$, 19.6, $20.0\left(2 \times \mathrm{CH}_{2}\right), 25.2\left(\mathrm{C}\left(\mathrm{CH}_{3}\right)_{3}\right), 28.2(\mathrm{CH})$, 31.2, $35.4(2 \times$

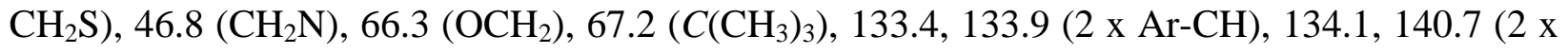


Ar-CH), 146.7, 150.0 (2 x Ar-C), 168.8, 176.5 (2 x C=O). MS, $m / z=422\left(\mathrm{M}^{+}\right), 340,214,198$, 173, 158, 130. HRMS: Calcd for $\mathrm{C}_{20} \mathrm{H}_{30} \mathrm{~N}_{4} \mathrm{O}_{4} \mathrm{~S}$ requires: $\mathrm{M}^{+}$422.1988; found: $\mathrm{M}^{+} 422.2013$.

N-tert-Butoxycarbonyl-S-[4-(benzotriazol-2-yl)butyl]cysteine ethyl ester (13k). Prepared according to the method used for 13a but using $\mathrm{NaH}$ (60\% in mineral oil; $0.04 \mathrm{~g}, 1.00 \mathrm{mmol}$ ) and $N$-tert-butoxycarbonyl-(R)-cysteine ethyl ester $5(0.20 \mathrm{~g}, 0.80 \mathrm{mmol})$, and allowing the mixture to warm to $20{ }^{\circ} \mathrm{C}$ over $10 \mathrm{~min}$ before the addition of $N^{2}$-(4-iodobutyl)benzotriazole $11 \mathbf{f}(0.97 \mathrm{~g}$, $3.22 \mathrm{mmol})$. The title compound was isolated as a yellow oil $(0.20 \mathrm{~g}, 61 \%), \mathrm{IR}\left(v_{\max }, \mathrm{NaCl} / \mathrm{cm}^{-1}\right)$ 3418, 2928, 1710, 1510, 1321, 1157. ${ }^{1} \mathrm{H}$ NMR (400 MHz, $\left.\mathrm{CDCl}_{3}\right), \delta_{\mathrm{H}} 1.23(3 \mathrm{H}, \mathrm{t}, J=7.1 \mathrm{~Hz}$, $\mathrm{CH}_{2} \mathrm{CH}_{3}$ ), 1.43 (9H, s, C( $\left.\left.\mathrm{CH}_{3}\right)_{3}\right), 1.66,2.21$ (each $\left.2 \mathrm{H}, \mathrm{m}, \mathrm{CH}_{2}\right), 2.49\left(2 \mathrm{H}, \mathrm{t}, J=7.2 \mathrm{~Hz}, \mathrm{CH}_{2} \mathrm{~S}\right.$ ), $2.58\left(2 \mathrm{H}, \mathrm{m}, \mathrm{CH}_{2} \mathrm{~S}\right), 4.15$ (2H, q, $\left.J=7.1 \mathrm{~Hz}, \mathrm{CH}_{2} \mathrm{CH}_{3}\right), 4.47(1 \mathrm{H}, \mathrm{m}, \mathrm{CH}), 4.73$ (2H, t, $J=6.2$ $\left.\mathrm{Hz}, \mathrm{CH}_{2} \mathrm{~N}\right), 5.28(1 \mathrm{H}, \mathrm{br}, \mathrm{NH}), 7.357 .84$ (each $\left.2 \mathrm{H}, \mathrm{m}, \mathrm{Ar}-\mathrm{H}\right) .{ }^{13} \mathrm{C}$ NMR $\left(100 \mathrm{MHz}, \mathrm{CDCl}_{3}\right), \delta_{\mathrm{C}}$ $9.9\left(\mathrm{CH}_{3}\right), 24.9\left(\mathrm{C}\left(\mathrm{CH}_{3}\right)_{3}\right), 27.0,29.1\left(2 \times \mathrm{CH}_{2}\right), 30.3(\mathrm{CH}), 31.0,40.2\left(2 \times \mathrm{CH}_{2} \mathrm{~S}\right), 45.4\left(\mathrm{CH}_{2} \mathrm{~N}\right)$,

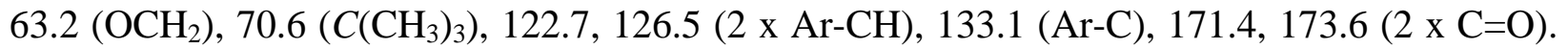
MS, $m / z=423\left(\mathrm{MH}^{+}\right), 340,214,184,173,158,130$. HRMS: Calcd for $\mathrm{C}_{20} \mathrm{H}_{30} \mathrm{~N}_{4} \mathrm{O}_{4} \mathrm{~S}: \mathrm{MH}^{+}$ 423.2060; found: $\mathrm{MH}^{+} 423.2063$.

\section{$N$-tert-Butoxycarbonyl-S-[2-(benzimidazol-1-yl)ethyl]cysteine (14)}

$N$-tert-Butoxycarbonyl-S-[2-(benzimidazol-1-yl)ethyl]cysteine ethyl ester 13d (0.5 g, 1.28 $\mathrm{mmol})$ in THF:methanol (50 mL, 3:1 v/v) was cooled in an ice bath, then $\mathrm{LiOH}$ solution (1M; $250 \mathrm{~mL}$ ) was then added, the mixture allowed to warm to $20^{\circ} \mathrm{C}$ and stirred for a further $8 \mathrm{~h}$. The organic solvents were removed under reduced pressure, the water layer acidified by hydrochloric acid (2M) to $\mathrm{pH} 4$ and the product extracted with EtOAc (3 x $20 \mathrm{~mL})$. The combined extracts were dried $\left(\mathrm{MgSO}_{4}\right)$ and the solvent removed under reduced pressure to leave the title compound as a white solid (0.45 g, 97\%), ( $\left.v_{\max }, \mathrm{NaCl} / \mathrm{cm}^{-1}\right) 3412$ (br), 2925, 1711, 1514, 1368, 1256, 1160. ${ }^{1} \mathrm{H}$ NMR (250 MHz, $\left.\mathrm{CDCl}_{3}\right), \delta_{\mathrm{H}} 1.43\left(9 \mathrm{H}, \mathrm{s}, \mathrm{C}\left(\mathrm{CH}_{3}\right)_{3}\right), 3.04-3.08\left(4 \mathrm{H}, \mathrm{m}, \mathrm{CH}_{2} \mathrm{SCH}_{2}\right), 4.52(1 \mathrm{H}$, m, CH), $4.67\left(2 \mathrm{H}, \mathrm{t}, J=7.2 \mathrm{~Hz}, \mathrm{CH}_{2} \mathrm{~N}\right), 5.64(1 \mathrm{H}, \mathrm{m}, \mathrm{NH}), 7.50-7.52(4 \mathrm{H}, \mathrm{m}, \mathrm{Ar}-\mathrm{H}), 7.96(1 \mathrm{H}$, s, $\mathrm{CH}=\mathrm{N})$. HRMS: Calcd for $\mathrm{C}_{17} \mathrm{H}_{23} \mathrm{~N}_{3} \mathrm{O}_{4} \mathrm{~S}$ requires: $\mathrm{MH}^{+}$366.1482; found: $\mathrm{MH}^{+}$366.1458.

\section{S-[2-(Benzimidazol-1-yl)ethyl]cysteine ethyl ester (15)}

$N$-tert-Butoxycarbonyl-S-[2-(benzimidazol-1-yl)ethyl]cysteine ethyl ester 13d (0.5 g, 1.28 mmol) was added to TFA: $\mathrm{CH}_{2} \mathrm{Cl}_{2}(50 \mathrm{~mL}, 1: 1 \mathrm{v} / \mathrm{v})$ and the mixture stirred for $2 \mathrm{~h}$. Diisopropylethylamine: $\mathrm{CH}_{2} \mathrm{Cl}_{2}(50 \mathrm{~mL}, 5: 95 \mathrm{v} / \mathrm{v})$ was then added to neutralise the acid. The solvents were removed under reduced pressure to give the crude amine that was chromatographed through a short silica column to give the title compound $(0.37 \mathrm{~g}, 86 \%)$, IR $\left(v_{\max }, \mathrm{NaCl} / \mathrm{cm}^{-1}\right)$ 3415, 2981, 1766, 1475, 1371. ${ }^{1} \mathrm{H} \mathrm{NMR}\left(250 \mathrm{MHz}, \mathrm{CDCl}_{3}\right), \delta_{\mathrm{H}} 1.22(3 \mathrm{H}, \mathrm{t}, J$ $\left.=7.1 \mathrm{~Hz}, \mathrm{CH}_{2} \mathrm{CH}_{3}\right), 3.07-3.09\left(4 \mathrm{H}, \mathrm{m}, \mathrm{CH}_{2} \mathrm{SCH}_{2}\right)$, $4.18\left(2 \mathrm{H}, \mathrm{q}, J=7.1 \mathrm{~Hz}, \mathrm{CH}_{2} \mathrm{CH}_{3}\right) 4.35(1 \mathrm{H}$, t, $J=5.6 \mathrm{~Hz}, \mathrm{CH}), 4.66\left(1 \mathrm{H}, \mathrm{t}, J=6.5 \mathrm{~Hz}, \mathrm{CH}_{2} \mathrm{~N}\right), 6.11\left(2 \mathrm{H}, \mathrm{br}, \mathrm{NH}_{2}\right), 7.49-7.63(4 \mathrm{H}, \mathrm{m}, \mathrm{Ar}-\mathrm{H})$, 7.95 (1H, s, $\mathrm{CH}=\mathrm{N})$. HRMS: Calcd for $\mathrm{C}_{14} \mathrm{H}_{19} \mathrm{~N}_{3} \mathrm{O}_{2} \mathrm{~S}$ : $\mathrm{MH}^{+}$294.1271; found: $\mathrm{MH}^{+}$294.1247. 


\section{Acknowledgements}

We acknowledge a studentship from Loughborough University (L. J. C.), and some MS data from the National Mass Spectrometry Service Centre, Swansea University.

\section{References and Notes}

1. Jones, R. C. F.; Berthelot, D. J. C.; Iley, J. N. Tetrahedron 2001, 57, 6539.

2. Jones, R. C. F.; Berthelot D. J. C.; Iley, J. N. Arkivoc 2007, (xi), 73.

3. Howarth, N. M.; Wakelin, L. P. G. J. Org. Chem. 1997, 62, 5441.

4. For an introduction to PNAs, see for example: Peptide Nucleic Acids: Protocols and Applications; Nielsen, P. E. Ed; Horizon Bioscience: Wymondham, 2004.

5. See, for example: Adlington, R. M.; Baldwin, J. E.; Catterick, D.; Pritchard, G. J. J. Chem. Soc., Perkin Trans. 1 1999, 855.

6. For O-linked (serine-based) heterocyclic amino acids assembled via a different strategy, see, for example: Garner, P.; Dey, S.; Huang, Y. in Pseudo-Peptides in Drug Discovery; Nielsen, P. E. Ed; Wiley-VCH: Weinheim, 2004; p 193.

7. For heterarylalanine derivatives from serine via Mitsunobu coupling to nucleobases, see: Guo, H.-M.; Wu, Y.-Y.; Niu, H.-Y.; Wang, D.-C.; Qu, G.-R. J. Org. Chem. 2010, 75, 3863.

8. Compound 12a showed just two methylene signals in the ${ }^{1} \mathrm{H}$ NMR spectrum, integral ratio 2:1 with the more intense signal as a downfield shifted triplet, $\delta_{\mathrm{H}} 4.90$, suggesting a symmetrical (quaternary salt) structure. Likewise, compound $\mathbf{1 2 b}$ showed two methylene signals, ratio 1:1, with one downfield triplet, $\delta_{\mathrm{H}}$ 4.57. In both cases, MS data indicated a dimeric salt with ions observed corresponding to $\mathrm{M}^{2+}$ and to $[\mathrm{M}-\mathrm{H}]^{+}$.

9. For a related example of a double salt from alkylation of benzimidazole with a 1,3dihalopropane, see: Murphy, J. A.; Khan, T. A.; Zhou, S.-z.; Thomson, D. W.; Mahesh, M. Angew. Chem. Int. Ed. Engl. 2005, 44, 1356.

10. Sugano, H.; Miyoshi, M. J. Org. Chem. 1976, 41, 2352.

11. Commercial $N$-tert-butoxycarbonyl-(RS)-homocysteine methyl ester and $N^{1}$-(2iodoethyl)benzimidazole $9 \mathbf{d}$ reacted under the $\mathrm{NaH}$ protocol to give the corresponding amino acid derivative in $57 \%$ yield.

12. Cruikshank, K. A.; Jiricny, J.; Reese, C. B. Tetrahedron Lett. 1984, 25, 681.

13. Díez-Barra, E.; de la Hoz, A.; Sánchez-Migallón, A.; Tejeda, J. Heterocycles 1992, 34, 1365.

14. Märky, M.; Schmid, H.; Hansen, H. J. Helv. Chim. Acta 1979, 62, 2129.

15. Krollpfeiffer, F.; Pötz, H.; Rosenberg, A. Chem. Ber. 1938, 71, 596.

16. Hirschberg, E.; Gellhorn, A.; Gump, W. S. Cancer Res. 1957, 17, 904.

17. Davies, R. G.; Gibson, V. C.; Hursthouse, M. B.; Light, M. E.; Marshall, E. L.; North, M.; Robson, D. A.; Thompson, I.; White, A. J. P.; Williams, D. J.; Williams, P. J. J. Chem. Soc., Perkin Trans. 1 2001, 3365. 
18. Shimidzu, T; Kanou, T.; Murakami, A.; Yamana, K.; Inagaki, H.; Donkai, N. J. Chem. Research (S) 1980, 376, (M) 4668. 